\title{
Electron beam guiding by a laser Bessel beam
}

\author{
Levi Schächter® $\circledast^{1, *}$ and W. D. Kimura $\odot^{2}$ \\ ${ }^{1}$ Technion-Israel Institute of Technology, Haifa 32000, Israel \\ ${ }^{2}$ STI Optronics, Inc., Bellevue, Washington 98005, USA
}

(Received 7 April 2020; accepted 15 June 2020; published 3 August 2020)

\begin{abstract}
We investigate the dynamics of electrons counterpropagating along a radially polarized optical Bessel beam (OBB). (i) It is shown that a significant fraction of the electrons can be transversally trapped by the OBB even in the case of "unmatched" injection. Moreover, (ii) these transversally trapped particles (TTPs) can be transported without loss along many thousands of wavelengths. As long as there is full longitudinal overlap between the electrons and laser pulse, this transport distance is limited only by the length of the OBB region. (iii) The unique profile of the transverse field components facilitates guiding either azimuthally symmetric pencil beams or annular beams. Space charge tends to totally suppress the annular beams, and it reduces the amount of charge trapped on axis for pencil beams. (iv) Assessment of the emittance of the TTPs alone reveals typical values of $10-50 \mathrm{pm}$. In fact, our simulations indicate if we trace the emittance of those particles that are trapped from the input to the output of the OBB, we find that this emittance is conserved. (v) We developed an analytic model whereby we average over the fast oscillation associated with the counterpropagating electrons and OBB. The resulting Hamiltonian has a Bessel potential $J_{1}^{2}(u)$, which, when operated in the linear regime near equilibrium, causes rotation of the phase space. A Kapchinskij-Vladimirskij beam-envelope equation is derived including space-charge and emittance effects. Relying on conservation of the longitudinal canonical momentum, the energy spread in the interaction region is determined in terms of the OBB intensity and the electron energy.
\end{abstract}

DOI: 10.1103/PhysRevAccelBeams.23.081301

\section{INTRODUCTION}

Guiding charged particles in vacuum along significant distances plays a pivotal role in many systems such as radiation sources, accelerators, and electron microscopes. Essentially, there are three types of transport systems: electrostatic systems are used primarily in electron microscopes and magnetostatic elements are used in high-energy accelerators. In a small, but by no means less important fraction, radio-frequency quadrupoles are used to both transport and accelerate either electrons or ions. In the present study, we demonstrate that a laser Bessel beam can transport efficiently a counterpropagating electron beam in vacuum. This may provide a highly useful alternative means for guiding electrons, especially for situations where it may be undesirable or impractical to have relatively large structures, such as magnets or metal components, near the electron beam. An example might be ultracompact electron

\footnotetext{
*Corresponding author. levis@technion.ac.il

Published by the American Physical Society under the terms of the Creative Commons Attribution 4.0 International license. Further distribution of this work must maintain attribution to the author(s) and the published article's title, journal citation, and DOI.
}

beam applications where conventional magnetic or electrostatic focusing cannot be used.

The notion of using optical beams to manipulate the trajectory of electrons is certainly not new. For example, there are various ways high-energy electrons can be focused with optical beams that rely on nearby structures $[1,2]$. The fields created within two colliding laser pulse can change the energy of subrelativistic electrons [3]. Optical micromanipulation of micron-sized particles using Bessel optical beams has been demonstrated [4]. What distinguishes our 8scheme from these other methods are (i) it occurs in free space with no nearby structures, (ii) it is designed to guide relativistic electrons, and (iii) it can be easily scaled to long interaction lengths, e.g., many meters.

Before we investigate the dynamics of the transport by an extended focus Bessel beam, it is useful to briefly review the essentials of acceleration of electrons by a focused beam in vacuum. The first hint regarding electron acceleration by a focused laser beam is in the work of Boivin and Wolf [5], where they investigated the electromagnetic field (Gaussian beam) in the vicinity of the focal point and found that a significant longitudinal electric field develops. In their words, "It seems plausible that such strong longitudinal fields could be used for accelerating charged particles. However, because of the considerable complexity of the field in the focal region, the practical feasibility of such a proposal must await a more detailed study." 
The next important step in our context was the configuration suggested by Steinhauer and Kimura [6]. Its essence was threefold: (i) a radially polarized laser beam; (ii) an aligned axicon focus that generates an extended focal region (Bessel beam); and (iii) the approximate matching of particle and laser phase velocities as both propagate in the same direction. They showed that acceleration is feasible provided the interaction region is terminated abruptly at both ends. A similar conclusion for a Gaussian beam was reached by Esarey, Sprangle, and Krall [7]. A couple of years later, the same group published another study [8] that is closely related to that presented by us in this study. It proposes a laser Bessel beam as a candidate to play a pivotal role in laser-driven accelerators. Because of the apparent similarity between these two studies, it is important to emphasize from the very beginning the differences between these studies and the work presented in this paper. The primary interest of these two studies was on creating a longitudinal accelerating force; thus, they both assumed copropagating electron and laser beams.

Our focus is on counterpropagating beams, where we are not interested in utilizing the longitudinal force, but rather we are using the fact that the particles will experience a rapidly oscillating transverse force. As we will show, this effective transverse force can guide the electron beam. Contrary to Ref. [8], which employs the paraxial approximation, we employ the exact solutions of $\mathrm{TM}_{01}$ in vacuum, although the finite cross section of the electron beam (e-beam) implies that only a small number of transverse Bessel "periods" are used in practice. In other words, we employ an ideal Bessel beam.

A different approach was adopted by Hora et al. [9]. They considered the feasibility of acceleration of individual electrons by Hermit-Gaussian laser beams, and it was shown that the transition from elastic to inelastic scattering occurs for a laser normalized amplitude $a=e E_{0} \lambda_{0} /$ $2 \pi m c^{2}>0.1$ and very small incident angle $\theta \ll \pi / 4$, where $e$ and $m$ represent the charge and the rest mass, respectively, of an electron, $c$ is the speed of light in vacuum, and $\lambda_{0}$ and $E$ represent the wavelength and the amplitude, respectively, of the laser. Moreover, later it was demonstrated in simulations [10] that, for an initial electron energy of $26 \mathrm{MeV}$ and some stringent initial conditions (among them $a \geq 100$ ), emerging $1.5 \mathrm{GeV}$ electrons are feasible. This is in comparison with $32 \mathrm{MeV}$ electrons for the case of inelastic scattering.

Further support of this result was provided 3 years later by Wang et al. [11], whereby the characteristics and essential conditions under which an electron in a vacuum and a laser beam can undergo a capture and acceleration scenario (CAS) were shown. They confirmed that, if $a \simeq 100$, the electron can be captured, primarily longitudinally, and violently accelerated to energies of the order of $1 \mathrm{GeV}$, corresponding to an effective acceleration gradient $1 \mathrm{TV} / \mathrm{m}$. As in the case of Ref. [2], the net energy gain is facilitated by the finite extent of the interaction and the momentary capture of the electron over this extended length. Implied in this work, as it pertains to this paper, is that CAS is enabled by the fact that diffraction of the focused laser beam leads to a significant component of its spectrum having a phase velocity equal to or slower than $c$.

To conclude this brief overview, at the high laser intensities involved, radiation-reaction effects on electron beams were considered [12]. Provided $a \gg 1$ and the initial relativistic factor $\gamma_{\text {ini }} \gg 1$, the dynamics divides into three regimes: (i) For relatively low-energy electrons, radiation damping effects are negligible. (ii) At higher electron energies, but still $2 \gamma_{\text {ini }}<a$, the damping alters the final displacement and the net energy change of the electron. (iii) For $2 \gamma_{\text {ini }}>a$, the radiation-reaction induces longitudinal electron trapping. This trapping process is stable with respect to the spatial properties of the electron beam and results in a significant energy loss of the electrons. In our case, we aim to transversally confine the beam with the lowest possible laser power; therefore, we assume that the radiation reaction is negligible.

Our idea stems from a previous concept [13] of generating x-ray radiation similar to channeling radiation in crystals, but in vacuum, by counterpropagating a beam of electrons through an optical Bessel beam (OBB). For the proof of principle analysis, we initially imposed some very stringent constraints on the model in order to facilitate the analytic solution. Recently, after we alleviated most of the constraints, rather than investigating the spontaneous radiation emitted, we decided to examine more closely the trajectories of the test particles ignoring the spontaneous radiation. The essence of this investigation is presented here: We found the OBB is able to transport electrons over its entire extent (many thousands of laser wavelengths). The diffraction-free character of Bessel beams [14] means that Rayleigh range limits no longer apply and the length of the Bessel beam can be made, in principle, as long as desired by simply increasing the diameter of the laser beam being focused by the axicon. We also found that typical normalized transverse emittances of less than $100 \mathrm{pm}$ were calculated for the transversally trapped particles (TTPs). In fact, we found that their emittance is preserved along the interaction length.

To thoroughly investigate the processes involved, we developed an analytic model whereby we average over the fast oscillation associated with the counterpropagating electron and Bessel laser beam. The resulting Hamiltonian has a radial Bessel potential $J_{1}^{2}(u)$, which, when operated in the linear regime near equilibrium, causes rotation of the phase space. This allows us to show the analytic conditions needed for the emittance to be conserved. A KapchinskijVladimirskij beam-envelope equation is derived including space-charge and emittance effects. In the OBB, the beam radius at equilibrium is reconfigured according to the intensity of the OBB, electron energy, and space charge. Relying on conservation of the longitudinal canonical 
momentum, the energy spread in the interaction region is determined in terms of the OBB intensity and the electron energy. We show that there is an inherent advantage to using a counterpropagating electron beam.

\section{DESCRIPTION OF ANALYSIS CONFIGURATION}

Let us first generate a clear picture of the configuration we intend to investigate. We conceive a radially polarized annular "plane wave," which extends between $R_{\text {int }} \leq r \leq R_{\text {ext }}$, and is focused by an axicon lens such that the Poynting vector crosses the axis at an angle $\theta_{0} \ll \pi-$ see Fig. 1. Before the axicon lens, the radial electric field in vacuum is denoted by $E_{r, 0}$, such that the input power flowing into the system is approximately given by $P_{\text {in }}=$ $\left(1 / 2 \eta_{0}\right) E_{r, 0}^{2} \pi\left(R_{\mathrm{ext}}^{2}-R_{\mathrm{int}}^{2}\right)$ and the net interaction length is $L=\left(R_{\text {ext }}-R_{\text {int }}\right) / \sin \theta_{0} \simeq R_{\text {ext }} / \theta_{0}$. It is assumed that the pulse duration $\tau_{p}$ is longer than the time it takes a relativistic electron to traverse the interaction length $\tau_{p}>L / c$ and, further, that the axicon lens is exposed to a fluence $F_{\max }$ below its damage threshold [15] $F=P_{\text {in }} \tau_{p} /$ $\pi\left(R_{\text {ext }}^{2}-R_{\text {int }}^{2}\right)<F_{\max }$. We should note that if larger intensities are desired, then it is possible to form an azimuthally symmetric OBB without using an axicon by coherently combining a large number of linearly polarized laser beams whose polarization directions are arranged in a radial pattern. Furthermore, it is possible to create any order Bessel beams using a kinoform phase plate [16] combined with an axicon as experimentally demonstrated by Fan et al. [17].

On axis, the longitudinal electric field has an amplitude $E_{0}=E_{r, 0} \sin \theta_{0} \simeq E_{r, 0} \theta_{0}$ implying that, near the axis and within the $e$-beam $\left(0<r<R_{b}\right)$, the electromagnetic field components can be derived from the magnetic vector potential and the scalar electric potential, which together satisfy the Lorentz gauge:

$$
\begin{aligned}
& A_{z}^{(\mathrm{OBB})}=-\frac{E_{0}}{\omega_{0} \sin ^{2} \theta_{0}} J_{0}\left(\frac{\omega_{0}}{c} r \sin \theta_{0}\right) \sin \left[\omega_{0}\left(t+\frac{z}{c} \cos \theta_{0}\right)\right], \\
& \Phi^{(\mathrm{OBB})}=\frac{E_{0} c \cos \theta_{0}}{\omega_{0} \sin ^{2} \theta_{0}} J_{0}\left(\frac{\omega_{0}}{c} r \sin \theta_{0}\right) \sin \left[\omega_{0}\left(t+\frac{z}{c} \cos \theta_{0}\right)\right] .
\end{aligned}
$$

$\lambda_{0}=2 \pi c / \omega_{0}$ is the laser's wavelength in vacuum, and $\omega_{0}$ is the laser frequency. This is an exact solution of the wave equation up to radius $r<R_{\max }$, beyond which the radial wave has to satisfy Sommerfeld's radiation condition

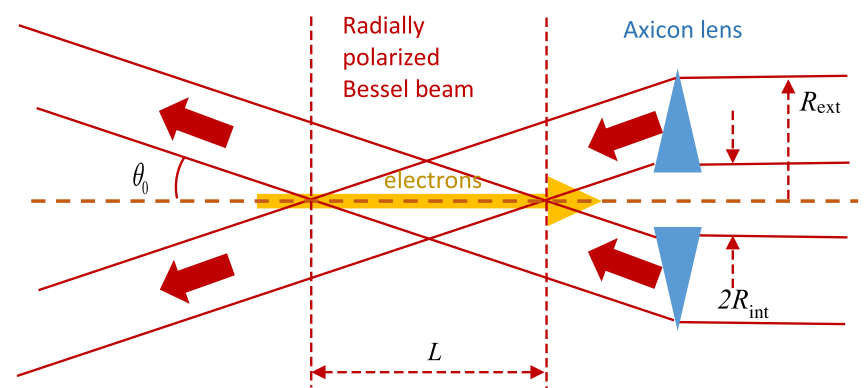

FIG. 1. An axicon lens converts a radially polarized annular $\left(R_{\text {int }}<r<R_{\text {ext }}\right)$ laser beam to an OBB on axis. The electrons counterpropagate against the Bessel beam. The laser beam propagates at an angle $\theta_{0}$ relative to the axis; the radial electric field before the axicon lens is $E_{r, 0}$, which translates on axis after the axicon to an amplitude $E_{z, 0} \equiv E_{0}=E_{r, 0} \sin \theta_{0}$. Not to scale.

$\left[J_{0}\left(\frac{\omega_{0}}{c} r \sin \theta_{0}\right) \rightarrow H_{0}^{(2)}\left(\frac{\omega_{0}}{c} r \sin \theta_{0}\right)\right]$. Throughout this study, we assume that the particles are much closer to the axis, namely, $r_{i} \leq r_{\max } \ll R_{\max } ; r_{i}$ is the radial coordinate of the $i$ th particle. Another aspect that is ignored is associated with the axicon lens, which has a small central hole in order to allow the electrons to traverse through the axicon without scattering. (Since the radially polarized laser beam impinging on the axicon is annular in shape, the central hole in the axicon will not significantly affect the laser beam.) Diffraction and Cerenkov radiation due to the passage of the electrons via this vacuum tunnel are ignored in this study. As clearly illustrated in Fig. 1, the OBB propagates in the opposite direction to the particles.

The single-particle trajectory can be determined by examining the equations of motion ignoring momentarily, for simplicity sake, space-charge and radiative effects:

$$
\begin{aligned}
\frac{d}{d \tau}\left(\gamma_{i} \dot{\bar{x}}_{i}\right) & =-\left(\cos \theta_{0}+\dot{\bar{z}}_{i}\right) \bar{x}_{i} \bar{U}_{i}, \\
\frac{d}{d \tau}\left(\gamma_{i} \dot{\bar{y}}_{i}\right) & =-\left(\cos \theta_{0}+\dot{\bar{z}}_{i}\right) \bar{y}_{i} \bar{U}_{i}, \\
\frac{d}{d \tau}\left(\gamma_{i} \dot{\bar{z}}_{i}\right) & =\left[\frac{1}{2} \frac{d}{d \tau}\left(\bar{x}_{i}^{2}+\bar{y}_{i}^{2}\right)\right] \bar{U}_{i}-\bar{V}_{i}, \\
\frac{d \gamma_{i}}{d \tau} & =-\cos \theta_{0}\left[\frac{1}{2} \frac{d}{d \tau}\left(\bar{x}_{i}^{2}+\bar{y}_{i}^{2}\right)\right] \bar{U}_{i}-\dot{\bar{z}}_{i} \bar{V}_{i}, \\
\frac{d \chi_{i}}{d \tau} & =2 \pi\left(1+\dot{\bar{z}}_{i} \cos \theta_{0}\right),
\end{aligned}
$$

where the various normalized quantities are defined next:

$$
\begin{aligned}
\tau & =c t / \lambda_{0}, \quad \bar{x}_{i}=x_{i} / \lambda_{0}, \quad \bar{y}_{i}=y_{i} / \lambda_{0}, \quad \bar{z}_{i}=z_{i} / \lambda_{0}, \quad \psi_{i}=2 \pi \sqrt{\bar{x}_{i}^{2}+\bar{y}_{i}^{2}} \sin \theta_{0}, \\
\bar{U}_{i} & =\pi \bar{E} J_{c}\left(\psi_{i}\right) \sin \left(\chi_{i}\right), \quad \bar{V}_{i}=\bar{E} J_{0}\left(\psi_{i}\right) \cos \left(\chi_{i}\right), \quad \bar{E}=e E_{0} \lambda_{0} / m c^{2}, \quad J_{c}(u) \equiv 2 J_{1}(u) / u .
\end{aligned}
$$


The first three equations represent the particles' momentum dynamics, the fourth the individual's particle energy dynamics, and the fifth the phase variation as experienced by the particle along the wave. In the equations of motion as formulated in Eq. (2), we omitted the fact that the force terms are exerted only in the region where the OBB exists, $0<z_{i}<L$; in the numerical simulations that follow, this effect is accounted for. In Appendix A, we describe in detail the steps that lead us to this set of equations. Before we proceed, it is important to point out that while $\gamma_{i}$, as well as the longitudinal momentum $\gamma_{i} \beta_{z, i}$ are affected by the Bessel beam, the phase of the counterpropagating relativistic electrons is virtually independent of the intensity of the optical field-see the last expression in Eq. (2), $\chi_{i}(\tau)=\chi_{i}(0)+2 \pi\left(1+\cos \theta_{0}\right) \tau \simeq \chi_{i}(0)+4 \pi \tau$. For this reason, we focus our attention on the transverse phase space.

\section{CONSTANTS OF MOTION}

From the fact that both potentials are azimuthally symmetric, we deduce that the angular momentum is conserved. Explicitly, using the two transverse equations of motion, we get

$$
\frac{d}{d \tau}\left(\bar{y}_{i} \gamma_{i} \dot{\bar{x}}_{i}-\bar{x}_{i} \gamma_{i} \dot{\bar{y}}_{i}\right)=0,
$$

which implies that the angular momentum is conserved; thus, its value at the input sets the angular momentum throughout the interaction region. Keeping in mind in cylindrical coordinates $\bar{x}_{i}=\bar{r}_{i} \cos \phi_{i}$ and $\bar{y}_{i}=\bar{r}_{i} \sin \phi_{i}$, we get by accounting for the $z$ component of the normalized angular momentum, $\mathcal{L}_{i} \equiv \gamma_{i}\left(\bar{x}_{i} \dot{\bar{y}}_{i}-\bar{y}_{i} \dot{\bar{x}}_{i}\right)$, that the azimuthal velocity is given by

$$
\dot{\phi}_{i}=\frac{\mathcal{L}_{i}}{\gamma_{i} \bar{r}_{i}^{2}}
$$

Further based on Eq. (4) and the definition of the relativistic factor, $\gamma_{i}^{-2} \equiv 1-\dot{\bar{r}}_{i}^{2}-\bar{r}_{i}^{2} \dot{\phi}_{i}^{2}-\dot{\bar{z}}_{i}^{2}$, we conclude that the latter is explicitly given by

$$
\gamma_{i}^{2}=\frac{1+\mathcal{L}_{i}^{2} \bar{r}_{i}^{-2}}{1-\dot{\bar{r}}_{i}^{2}-\dot{\bar{z}}_{i}^{2}} .
$$

In the simulations that follow, the angular momentum is taken to be zero $\left(\mathcal{L}_{i}=0\right)$, except if specified otherwise.

Another constant of motion is deduced from the other two equations of motion (for $\gamma_{i} \dot{\bar{z}}$ and $\gamma_{i}$ ). In both cases, we have the term $\dot{\bar{r}}_{i} \bar{r}_{i} \bar{U}_{i}$ on the right-hand side; therefore, after some straightforward steps, we obtain conservation of the canonical longitudinal momentum:

$\frac{d}{d \tau}\left[\gamma_{i}\left(\dot{\bar{z}}_{i}+\cos \theta_{0}\right)+\frac{\bar{E}}{2 \pi} \sin \left(\chi_{i}\right) J_{0}\left(2 \pi \bar{r}_{i} \sin \theta_{0}\right)\right]=0$.

At the end of the last section, we note that, for relativistic particles $\gamma_{i} \gg 1$, the phase of the particles is not affected by the intensity of the OBB. The conservation of canonical longitudinal momentum, as reflected in Eq. (6), indicates that, for a narrow pencil beam such that $J_{0}\left(2 \pi \bar{r}_{i} \sin \theta_{0}\right) \simeq 1$, the energy of the particle can be solved analytically. In Appendix B, we show that the initial energy spread $\Delta \gamma_{\text {ini }}^{2}$ increases in the interaction region according to the intensity of the OBB:

$$
\Delta \gamma_{\mathrm{ini}}^{2} \rightarrow \Delta \gamma^{2}=\Delta \gamma_{\mathrm{ini}}^{2}+\left[\frac{e E_{0} \lambda_{0} / m c^{2}}{2 \pi\left(1+\cos \theta_{0}\right)}\right]^{2}
$$

however, as the interaction ends, since the canonical longitudinal momentum is conserved, the energy spread will return to its original value, $\Delta \gamma_{\text {ini }}^{2}$.

Let us now return to the main theme of this section, constants of motion. The four equations of motion postulated in Eq. (2) can be reduced to three if we multiply the first three with the corresponding momentum, add all three, and subtract the fourth after being multiplied by $\gamma_{i}$. The result

$$
\frac{d}{d \tau}\left[\frac{1}{2}\left(\gamma_{i} \dot{\bar{x}}_{i}\right)^{2}+\frac{1}{2}\left(\gamma_{i} \dot{\bar{y}}_{i}\right)^{2}+\frac{1}{2}\left(\gamma_{i} \dot{\bar{z}}_{i}\right)^{2}-\frac{1}{2} \gamma_{i}^{2}\right]=0
$$

is a manifestation of the fact that the magnitude of the energy-momentum's 4-vector is constant. Evidently, we have the choice of using the fourth equation of motion to determine $\gamma_{i}$ or ignore the former and define $\gamma_{i}$ based on Eq. (8). As a result, the three degrees of freedom (three momenta components) are reduced to one by the two constants of motion specified above. With that in mind, the dynamics are determined by the radial motion alone:

$$
\begin{aligned}
\frac{d}{d \tau}\left(\gamma_{i} \dot{\bar{r}}_{i}\right)-\frac{\mathcal{L}_{i}^{2}}{\gamma_{i} \bar{r}_{i}^{3}}= & -\frac{\bar{E}}{\sin \theta_{0}} J_{1}\left(2 \pi \bar{r}_{i} \sin \theta_{0}\right)\left(\cos \theta_{0}+\dot{\bar{z}}_{i}\right) \\
& \times \sin \left(\chi_{i}\right) .
\end{aligned}
$$

With the equations of motion simplified, one needs to specify the important measure of transverse confinement, namely, the emittance. Assuming that the radial coordinate is based on Eq. (8), the transverse normalized emittance may be assessed by adopting the definition originally suggested by Lapostolle [18]:

$$
\varepsilon_{x}=4 \pi \lambda_{0} \sqrt{\left\langle\left[\bar{x}_{i}(\tau)\right]^{2}\right\rangle_{i}\left\langle\left[\dot{\bar{x}}_{i}(\tau)\right]^{2}\right\rangle_{i}-\left\langle\bar{x}_{i}(\tau) \dot{\bar{x}}_{i}(\tau)\right\rangle_{i}^{2}} .
$$

\section{LINEAR REGIME}

For establishing the beam stability, we examine the case of zero longitudinal angular momentum, $\mathcal{L}_{i}=0$, which allows electrons to move on axis; otherwise, radially the acceleration diverges-see Eq. (9). In this case, we observe for certain values of $\bar{r}_{i}$ that the radial force vanishes and the particles' dynamics in the radial direction is as if no OBB is present. For this equilibrium state to happen, the radius $\bar{r}_{\mathrm{eq}}$ has to satisfy 


$$
2 \pi \bar{r}_{\mathrm{eq}, s} \sin \theta_{0}=p_{1, s},
$$

where $p_{1, s}$ are the relevant zeros of the first-order Bessel function $J_{1}\left(p_{1, s}\right) \equiv 0$ and $s$ is an integer $s=0,1,2 \ldots$ with its limit determined by the geometric radius of the electron beam and the angle of the laser OBB, i.e., $p_{1,0}=0$, $p_{1,1}=3.832, p_{1,2}=7.016, p_{1,3}=10.173 \ldots$ Note that in the longitudinal direction, according to the conservation of the canonical longitudinal momentum in the $z$ direction, the electron oscillates. Furthermore, we can assume that a small transverse oscillation $\left(\delta \bar{r}_{s, i}\right)$ around the equilibrium does occur, namely, $\bar{r}_{i}=\bar{r}_{\mathrm{eq}, s}+\delta \bar{r}_{s, i}$; therefore, ignoring the longitudinal oscillation as well as the energy modulation, $\gamma_{i}(\tau)=\gamma_{i}(0) \equiv \gamma_{i, 0}$, for relativistic particles $\left(\bar{p}_{z, i}, \gamma_{i} \gg 1\right)$, the radial equation of motion simplifies to read

$$
\left[\frac{d^{2}}{d u^{2}}+\alpha_{s, i} \sin \left(\chi_{0, i}+u\right)\right] \delta \bar{r}_{s, i}=0
$$

where $\quad \alpha_{s, i} \equiv \bar{E} J_{0}\left(p_{1, s}\right)\left[4 \pi \gamma_{i, 0} \cos ^{2}\left(\theta_{0} / 2\right)\right]^{-1} \quad$ and $\quad u \equiv$ $4 \pi \cos ^{2}\left(\theta_{0} / 2\right) c t / \lambda_{0}$. This is the Mathieu equation that can have periodic (thus stable) solutions. In Appendix C, we show that a stable solution is possible for $s=1$ if $\alpha_{1, i}=$ $\left[J_{0}\left(p_{1,0}\right) \bar{E}\right]\left[\gamma_{0, i} 4 \pi \cos ^{2}\left(\theta_{0} / 2\right)\right]^{-1} \leq 0.454$ or, explicitly,

$$
\gamma_{0} \geq 0.175 \frac{e E_{0} \lambda_{0}}{m c^{2}}
$$

For most practical regimes of interest, the right-hand side of Eq. (12) is much smaller than one; therefore, for relativistic electrons, this constraint is always satisfied. This is the first important result of this study, since it indicates that, within the framework of a linear approximation, electrons moving in an OBB can follow stable trajectories which can form a pencil beam $(s=0)$ or annular beam $(s>0)$.

\section{DYNAMICS WITHOUT SPACE CHARGE}

The important result mentioned above and analyzed subject to a linear approximation is next investigated numerically relying on the exact set of equations in Eq. (2) and employing the set of parameters specified in Table I. Figure 2 shows the transverse phase space when the space-charge effect is ignored. As reference, we show the initial $(z=0)$ phase space represented by red circles in both frames. Each circle represents a microparticle that contains many electrons that are "glued together" all along the interaction length. It is tacitly assumed that, by splitting the entire beam into $N_{\text {mp }}$ microparticles, the characteristic features of the entire ensemble will be preserved. It should be made crystal clear that, while we focus here on the transverse dynamics, the latter is solved self-consistently with the longitudinal dynamics. And in the longitudinal direction we consider one period of the exerted field, since at a given location, during the next period of time,
TABLE I. The default values of the parameters used in the various simulations.

\begin{tabular}{lcc}
\hline \hline$\lambda_{0}=1.064[\mu \mathrm{m}]$ & $\bar{E}=1.2 \times 10^{-5}$ & $\gamma_{0}=20$ \\
$E_{r, 0}=33[\mathrm{MV} / \mathrm{m}]$ & $L \simeq 1.0[\mathrm{~m}]$ & $\left|\gamma_{i}(0) \dot{\bar{x}}_{i}(0)\right| \leq 10^{-4}$ \\
$S_{z, 0}=147.4[\mathrm{MW} / \mathrm{cm}]$ & $F \simeq 1.0\left[\mathrm{~J} / \mathrm{cm}^{2}\right]$ & $\left|\gamma_{i}-\gamma_{0, i}\right| \leq 10^{-3} \gamma_{0, i}$ \\
$\theta_{0}=10(\pi / 180)$ & $R_{b} \simeq 5 \lambda_{0}$ & $N_{\mathrm{mp}}=360$ \\
\hline \hline
\end{tabular}

the process repeats itself. It is for this reason that we consider one period of the exerted field that contains $N_{\text {mp }}$ microparticles [19-20]. As a typical example, we can conceive a 3-mm-long pulse of $10^{9}$ electrons, and the exerted force has a $3 \mu \mathrm{m}$ period; therefore, in one period, there are $10^{6}$ electrons. These can be divided into 1000 microparticles, each one containing 1000 electrons. Throughout all simulations that follow, the relative error, defined based on Eq. (8), did not exceed $10^{-9} \%$.

In Fig. 2(a), the blue circles show how the transverse phase space evolved after half a meter. Several facts are evident:
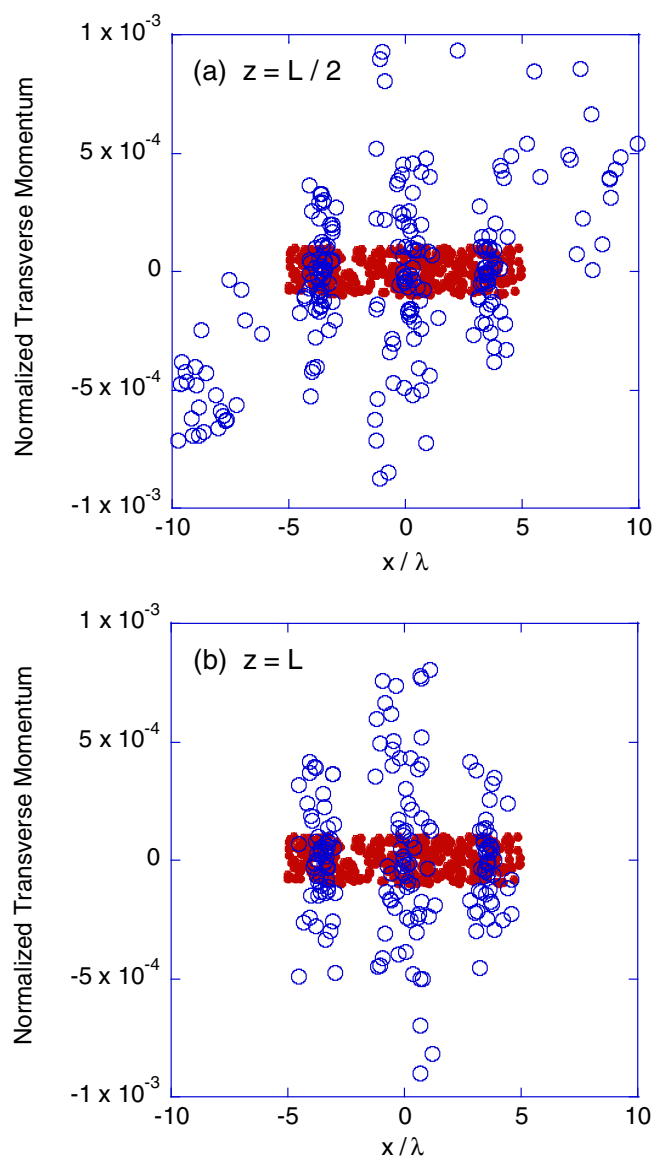

FIG. 2. Phase space in the space-charge-free case. The trapping of particles in the vicinity of the equilibrium points is evident: $\left|\bar{x}_{\text {eq }}\right| \sim 0$ and $\left|\bar{x}_{\text {eq }}\right| \sim 3.512$. The initial beam's phase space does not cover the third equilibrium $\left(\left|\bar{x}_{\text {eq }}\right| \sim 6.43\right)$ since $|\bar{x}|<5$; therefore, electrons are not trapped in this state. (a) At $z=L / 2$. (b) At $z=L$. 
Already at this stage, part (20\%) of the microparticles (representing the electrons) are trapped $\left[J_{1}\left(\bar{x}_{\text {eq }}\right) \equiv 0\right]$ on axis $\bar{x}_{\mathrm{eq}} \simeq 0$ forming a "pencil beam." Another fraction (29\%) is trapped in the vicinity of the second zero of the Bessel function corresponding to $\left|\bar{x}_{\text {eq }}\right| \simeq 3.512$, indicating the generation of an "annular beam." (The parameters for this particular example were chosen to illustrate how it is possible for both a pencil and an annular beam to be guided simultaneously by the OBB. Although actual applications would typically guide only one type of beam, this particular example will be useful in understanding the effects of space charge as discussed shortly.) A third group is not trapped, but rather they slowly "diffuse" away from the center. Not shown is a fourth group of electrons that were scattered in the first few centimeters of the interaction.

In Fig. 2(b), only the trapped electrons (49\%) are left, and this is a representative picture for the last quarter of the interaction region. Note that there are no trapped microparticles in the third equilibrium point $\left|\bar{x}_{\text {eq }}\right| \simeq 6.43$. In fact, only microparticles that are initially in the vicinity of the equilibrium are trapped. For example, if we reduce (not
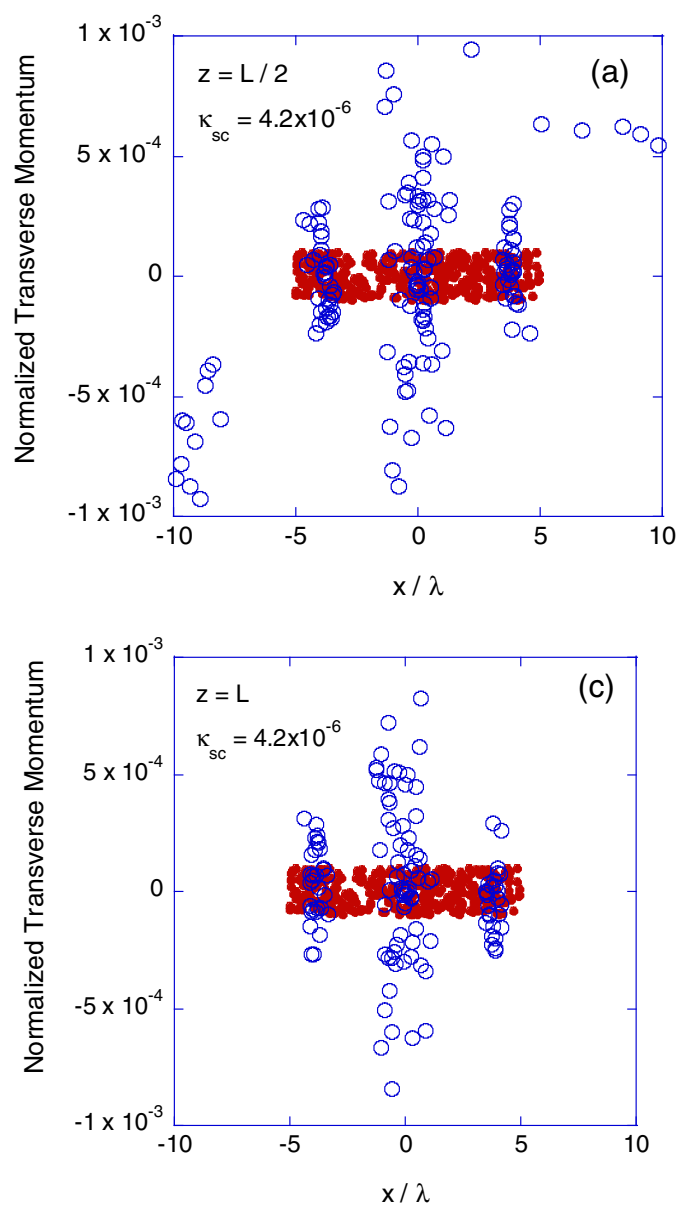

shown) the phase space at the input to be $\left|\bar{x}_{i}\right| \leq 2$, then no particles are trapped in the vicinity of the second equilibrium point. This is the second important result of this study, since it indicates that the OBB supports the generation of either a pencil or an annular beam for rather large amplitudes. Thus, the stable trajectories are not limited to the linear regime.

A third important result is associated with the emittance. Its value for the pencil beam at the output $(z=L)$ is $\varepsilon_{n} \simeq$ $53.2 \mathrm{pm}$. Tracing backwards to those electrons that made it to the end of the OBB and calculating their emittance at $z=L / 2$, we find that the emittance is $\varepsilon_{n} \simeq 53.5 \mathrm{pm}$, which clearly shows that the emittance of the TTPs is conserved for at least the second half meter. The slight deviation can be attributed to the usage of a relatively small number of microparticles (70) affecting the statistics.

\section{DYNAMICS WITH SPACE CHARGE}

For guiding a significant number of monoenergetic electrons, we need to confine them as much as possible on the scale of the OBB wavelength with the limit being the
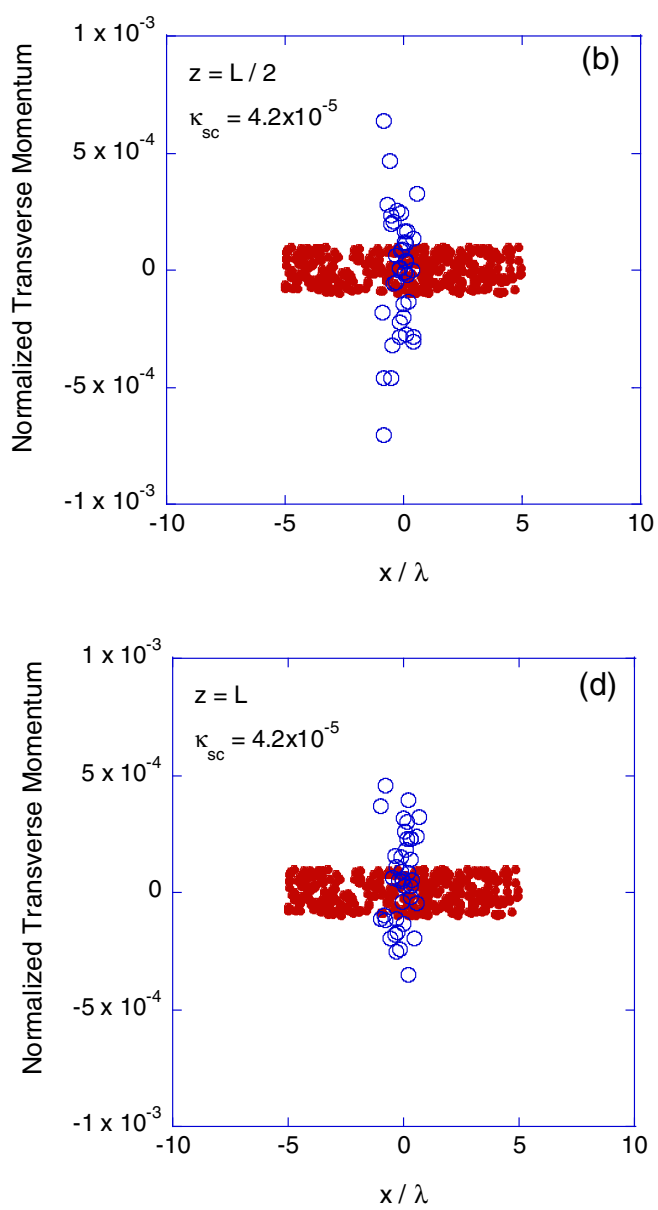

FIG. 3. (a) $\kappa_{\mathrm{SC}}=4.2 \times 10^{-6}, z=L / 2$. (c) $\kappa_{\mathrm{SC}}=4.2 \times 10^{-6}, z=L$. Space charge tends to reduce the trapping when comparing $\kappa_{\mathrm{sc}}=4.2 \times 10^{-6}$ with the zero space-charge case $\kappa_{\mathrm{sc}}=0$ as revealed in Fig. 2 . (b) $\kappa_{\mathrm{SC}}=4.2 \times 10^{-5}, z=L / 2$. (d) $\kappa_{\mathrm{SC}}=4.2 \times 10^{-5}$, $z=L$. Elevating the charge by one order of magnitude suppresses entirely the annular beam. 
repelling force the electrons exert on each other. When this force is greater than or equal to the radial force exerted by the OBB, the electron beam will tend to blow up. Our goal now is twofold: expand the equations of motion to account for the space-charge effect and assess the effect of the latter. The former is explained in detail in Appendix D, and in
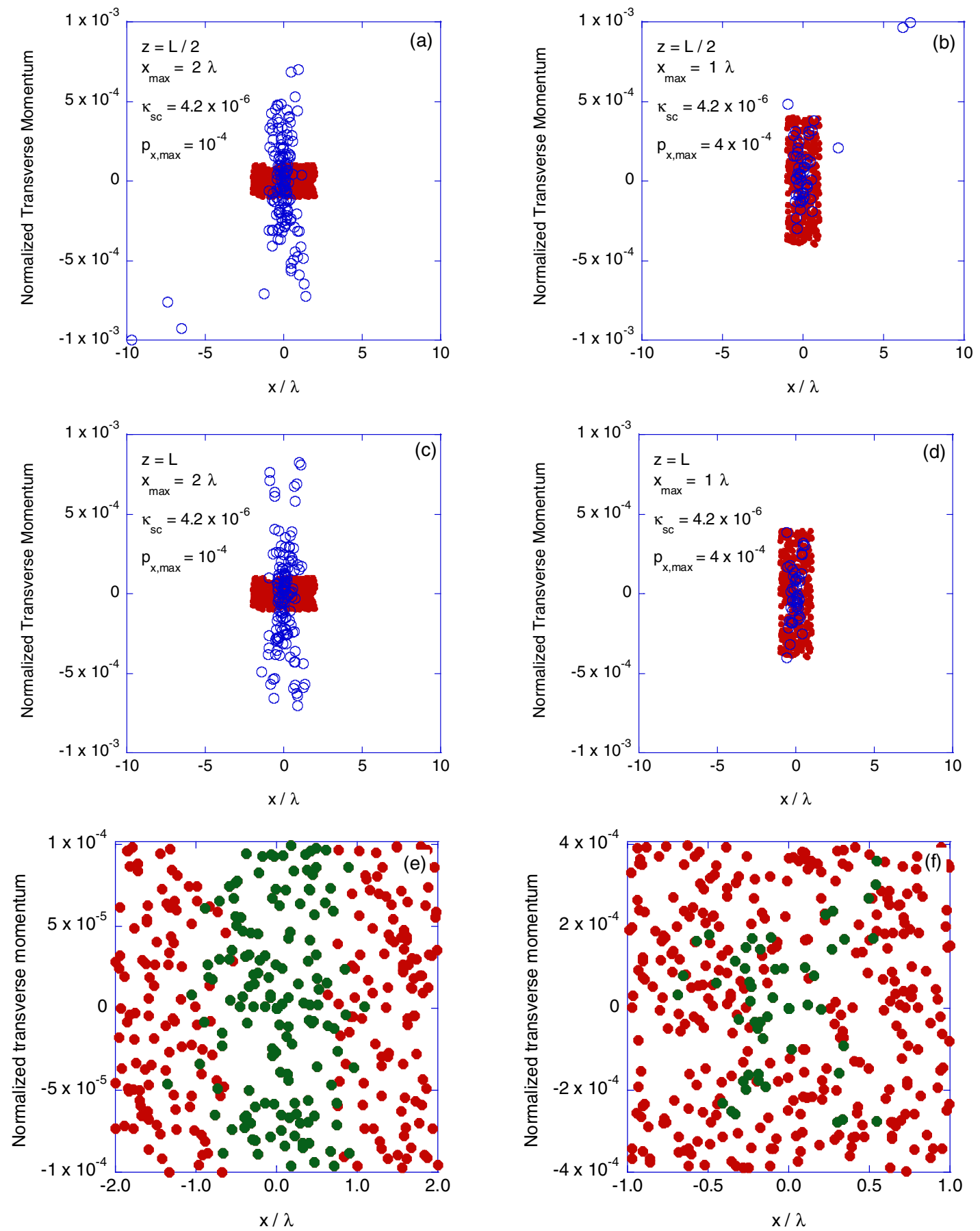

FIG. 4. Guiding of a pencil beam of electrons by OBB. (a) $\kappa_{\mathrm{SC}}=4.2 \times 10^{-6}, z=L / 2, p_{x \text {, max }}=10^{-4}$. (c) $\kappa_{\mathrm{SC}}=4.2 \times 10^{-6}, z=L$, $p_{x, \max }=10^{-4}$. The low space-charge $\left(\kappa_{\mathrm{sc}}=4.2 \times 10^{-6}\right)$ beam is significantly squeezed spatially, but the spread of the transverse momentum increases by almost one order of magnitude. The initial emittance is $0.1 \mathrm{~nm}$. (e) Phase space at the input is marked with red, whereas in green are labeled particles at the input that eventually become trapped and make it to the output. A well-defined region $\left(\left|\bar{x}_{i}\right| \leq 1\right)$ in the phase space is occupied by only those electrons that will become trapped, implying that the emittance of the trapped electrons is within a good approximation constant. (b),(d),(f) The same parameters as (a),(c),(e) except $\bar{x}_{\max }=1.0, \bar{p}_{x, \text { max }}=4 \times 10^{-4}$. Note that many fewer electrons are trapped, since the selection is more stringent, but the emittance of the trapped particles $(0.2 \mathrm{~nm})$ is significantly lower than that of the entire ensemble at the input $(1 \mathrm{~nm})$. 
what follows we discuss the main findings from our numerical investigation. In the framework of the approximation specified in Appendix D, space charge can be represented by a single parameter $\kappa_{\mathrm{sc}} \equiv 2 r_{\mathrm{el}} N_{\Delta}$, where $r_{\mathrm{el}}=2.8 \times 10^{-15}[\mathrm{~m}]$ is the classical radius of the electron and $N_{\Delta}$ represents the number of electrons per unit length in the direction of motion. In zero order, for stability, we require that the transverse force exerted by the OBB is dominant, which is shown in Appendix $\mathrm{D}$ to be equivalent to $\pi E_{0} \gg e N_{\Delta} / 4 \pi \varepsilon_{0} \lambda_{0} \gamma^{2}$ and is readily satisfied even at moderately relativistic energies, e.g., $\gamma \simeq 20$.

The first observation we make is that space-charge effects tend to suppress the annular beams: Figs. 3(a) and 3(c) correspond to a small space-charge parameter $\kappa_{\mathrm{sc}}=4.2 \times 10^{-6}$ at $z=L / 2$ and $z=L$, respectively. We observe that fewer electrons (36\% compared to $49 \%$ in the space-charge-less case in Fig. 2) are trapped and those trapped have a slightly larger spatial spread. Elevating the number of electrons by one order of magnitude, $\kappa_{\mathrm{sc}}=4.2 \times 10^{-5}$, as evident in Figs. 3(b) and 3(d), the annular beam is absent even in the middle of the interaction length. For the pencil beam, $11 \%$ of the particles are trapped and are transported over the 1-m-long interaction region. The emittance at the output $(z=L)$ of the pencil beam is $\varepsilon_{n} \simeq 45.1 \mathrm{pm}$; whereas, tracing backwards, the trapped electrons had an emittance of $\varepsilon_{n} \simeq 48.5 \mathrm{pm}$ at $z=L / 2$. This is for the low space-charge case. For the high space-charge case, the emittance is a factor of 2.5 lower, $\varepsilon_{n}(z=L) \simeq 17.4 \mathrm{pm}$ and $\varepsilon_{n}(z=L / 2) \simeq 21.1 \mathrm{pm}$. Although to a lesser extent, in this case, too, the emittance of the TTPs may be considered essentially constant in the second half of the interaction region. We should note that the effects of possible radiation loss have not been included in this analysis. Furthermore, there may be a self-filtering process occurring in which low emittance electrons tend to remain well guided, whereas higher emittance electrons tend to be lost during transit along the first half of the $1 \mathrm{~m}$ OBB length $\left(10^{6}\right.$ wavelengths).

Next, we focus our attention to a regime where only a pencil beam is guided. For this purpose, we limit the initial spatial spread of the electrons to $\left|\bar{x}_{i}\right| \leq 2$ and we consider the case of a weak space-charge effect $\left(\kappa_{\mathrm{sc}}=4.2 \times 10^{-6}\right)$; the initial momentum spread is $\left|\bar{p}_{x, i}\right| \leq$ $1 \times 10^{-4}$. Figures 4(a) and 4(c) show the phase-space distribution at $z=L / 2$ and $z=L$, respectively. These results reveal that, despite space-charge effects, there is no major change in the transverse phase space along most of the last half-meter guidance "tunnel" formed by the OBB [see Figs. 4(a) and 4(c)] and the TTPs are transported without loss (40\% of the initial particles are trapped). It is interesting to note that the electrons, which are guided along the second half of the interaction, have an emittance comparable with their value in the previous cases $\left[\varepsilon_{n}(z=L) \simeq 34 \mathrm{pm}\right.$ and $\left.\varepsilon_{n}(z=L / 2) \simeq 29.2 \mathrm{pm}\right]$. While clearly the phase space is squeezed spatially, along the
TABLE II. Emittance in the various regimes considered. In brackets is the percentage of the TTPs near the axis.

\begin{tabular}{lccc}
\hline \hline$\kappa_{\mathrm{sc}}$ & $\begin{array}{c}\varepsilon[\mathrm{pm}] @ \\
z=0\end{array}$ & $\begin{array}{c}\varepsilon_{\text {trapped }}^{\text {pencil }}[\mathrm{pm}] @ \\
z=L\end{array}$ & $\begin{array}{c}\varepsilon_{\text {trapped }}^{\text {pencil }}[\mathrm{pm}] @ \\
z=L / 2\end{array}$ \\
\hline 0 & 35.5 & $53.2(19.4 \%)$ & $53.5(19.4 \%)$ \\
$4.2 \times 10^{-6}$ & 35.5 & $45.1(19.2 \%)$ & $48.5(19.2 \%)$ \\
$4.2 \times 10^{-5}$ & 35.5 & $17.4(11.1 \%)$ & $21.1(11.1 \%)$ \\
$4.2 \times 10^{-6}$ & 14.4 & $34.4(39.7 \%)$ & $29.2(39.7 \%)$ \\
$4.2 \times 10^{-6}$ & 28.5 & $10(13.1 \%)$ & $10(13.9 \%)$ \\
\hline \hline
\end{tabular}

momentum coordinate its spread is almost one order of magnitude larger-yet the electrons remain trapped.

In Fig. 4(e), we see the transverse phase space at the input marked with red, whereas in green we labeled those particles at the input that eventually became trapped and made it all the way through to the output. Clearly, there is a well-defined region $\left(\left|\bar{x}_{i}\right| \leq 1\right)$ in the phase space occupied by only those electrons that will become trapped. This brings us to the fourth important result of this study that the emittance is not reduced, but rather it is conserved; in other words, the emittance of the trapped electrons at the input is within a good approximation constant along at least the second half of the interaction region.

The transverse phase space at the output of Fig. 4(c) leads us to examine the case when the initial transverse phase space is squeezed spatially $\left(\left|\bar{x}_{i}\right| \leq 1\right)$ but enlarged along the transverse momentum $\left|\bar{p}_{x, i}\right| \leq 4 \times 10^{-4}$ axissee Figs. 4(b) and 4(d). In the middle of the interaction region, there are very few (15\%) microparticles that remained untrapped [Fig. 4(b)], and this number diminishes at the end of the guiding process [Fig. 4(d)]. In this case, the emittance of the TTPs at the output is $10 \mathrm{pm}$. Figure 4(f) reveals that the relatively large transverse momentum spread as well as the space-charge effect makes the origin of the trapped particles more complex compared to the case in Fig. 4(e), but the emittance remains unchanged (10 pm). Again, in the framework of our numerical analysis, the emittance of the trapped particles seems to be conserved. In Table II, we summarize the normalized emittances in the various regimes.

\section{ANALYTIC MODEL: SLOWLY VARYING AMPLITUDE}

A deeper insight into the preceding numerical results may be obtained by developing a Hamiltonian that accounts for averaging over the fast oscillations associated with the electron and the wave propagating in opposite directions. In Appendix E, we show that the Hamiltonian associated with the radial motion $\rho_{i}=\alpha_{i} J_{1}\left(C_{i}\right) \sin \left(\chi_{i, 0}+\psi\right)+C_{i} \simeq C_{i}$ with the normalized time variable $\psi=2 \pi\left(c t / \lambda_{0}\right)(1+$ $\left.\cos \theta_{0}\right)$ and the normalized radius $\rho_{i}=2 \pi\left(r_{i} / \lambda_{0}\right) \sin \theta_{0}$, as well as the normalized coupling coefficient $\alpha_{i} \equiv\left(e E_{0} \lambda_{0} / m c^{2}\right)\left[2 \pi \gamma_{i, 0}\left(1+\cos \theta_{0}\right)\right]^{-1} \ll 1$, reads 


$$
\mathcal{H}=\frac{1}{2}\left(\frac{d C_{i}}{d \psi}\right)^{2}+\frac{1}{4} \alpha_{i}^{2} J_{1}^{2}\left(C_{i}\right)
$$

It contains a nonlinear potential $V\left(C_{i}\right)=\frac{1}{4} \alpha_{i}^{2} J_{1}^{2}\left(C_{i}\right) \simeq$ $\frac{1}{2}\left(\frac{\alpha_{i}^{2}}{8}\right) C_{i}^{2}$, which for small radii, $C_{i}=2 \pi\left(r_{i} / \lambda_{0}\right) \sin \theta_{0} \ll 1$, indicates that the transverse motion is stable and oscillates with a normalized frequency $\alpha_{i} 2^{-3 / 2}$ or in non-normalized units $\Omega_{i}=e E_{0} c / m c^{2} \gamma_{i, 0} 2^{3 / 2}$.

For large radii, there are stable oscillations near any zero of the potential well $J_{1}\left(p_{1, s}\right)=0$ - as already introduced in the context of Eq. (15). The first zero $\left(p_{1, s=0}=0\right)$ represents a pencil beam, whereas all the others $\left(p_{1, s>0}\right)$ represent annular beams.

\section{E-BEAM TRANSPORT}

We are now in position to examine three aspects of the $e$-beam transport by the OBB. First, we determine the maximum transverse momentum allowed in order to facilitate trapping. Second, by means of a transport matrix formulation, it is shown that the transverse emittance is conserved, and, third, we develop the equivalent Kapchinskij-Vladimirskij [21] equation that determines the equilibrium radius of the (pencil) beam and the dynamics of small perturbation around this equilibrium.

\section{A. Maximum transverse momentum}

From the perspective of the oscillation's amplitude, it is limited to the range between two adjacent maxima, $\dot{J}_{1}\left(\dot{p}_{1, s}\right) \equiv 0$, of the potential $\dot{p}_{1, s \geq 0} \leq C \leq \dot{p}_{1, s+1}$; therefore, according to the definition of the Hamiltonian [Eq. (15)] the radial velocity is limited to

$$
\begin{aligned}
0 & \leq\left(\frac{d C_{i}}{d \psi}\right)^{2}=2 \mathcal{H}_{i}-\frac{1}{2} \alpha_{i}^{2} J_{1}^{2}\left(C_{i}\right) \leq\left|\frac{d C_{i}}{d \psi}\right|_{\psi=0}^{2} \\
& +\frac{1}{2} \alpha_{i}^{2}\left\{J_{1}^{2}\left[C_{i}(\psi=0)\right]-J_{1}^{2}\left(\dot{p}_{1, s+1}\right)\right\},
\end{aligned}
$$

implying for the worst case scenario for a pencil beam $\left[C_{i}(\psi=0)=0\right]$ that the (normalized) radius should not exceed the location of the largest maximum $\left(\dot{p}_{1, s}\right)$ of the potential. For example, in the case of a pencil beam, the Bessel function has a maximum: $J_{1}^{2}(u) \leq J_{\max }^{2} \equiv 0.339$ ( $u \simeq 1.841)$; therefore, the limit on the initial radial motion allowed is $\left|\left(d C_{i} / d \psi\right)_{\psi=0}\right|<(1 / 2) \alpha_{i}\left|J_{1}\left(\dot{p}_{1,1}\right)\right|$ or, explicitly, $\left|\gamma \beta_{r}\right|_{t=0} \leq 0.065\left(e E_{0} \lambda_{0} / m c^{2}\right)\left(1 / \sin \theta_{0}\right)$. Keeping in mind that based on Fig. 1 we may deduce that $P_{\text {in }}=$ $\left(1 / 2 \eta_{0}\right) E_{r, 0}^{2} \pi R_{\mathrm{ext}}^{2}$ and $E_{r, 0}=E_{0} / \sin \theta_{0}$, thus we get

$$
\left|\gamma \beta_{r}\right|_{t=0}<0.065 \sqrt{\frac{2 \eta_{0} P_{\mathrm{in}}}{\left(m c^{2} / e\right)^{2}} \frac{\lambda_{0}^{2}}{\pi R_{\mathrm{ext}}^{2}}},
$$

implying that the upper limit of the transverse momentum is set by the input energy flux before the axicon lens (e.g.,
$F=1 \mathrm{~J} / \mathrm{cm}^{2}$ ). For $\lambda=1 \mu \mathrm{m}, L=1 \mathrm{~m}$, and $R_{\text {ext }}=10 \mathrm{~cm}$, we find $\left|\gamma \beta_{r}\right|_{t=0}<6 \times 10^{-3}$, which implies that any electrons entering the $\mathrm{OBB}$ region with a radial velocity greater than this value will not be trapped.

\section{B. Emittance conservation}

Let us examine the emittance as electrons are oscillating near one of the zeros of the potential $p_{1, s}: J_{1}\left(p_{1, s}\right) \equiv 0$, $s=0,1,2, \ldots$. Consider a linearized solution of the form $C_{i} \simeq p_{1, s}+\delta C_{s, i}$, and then the trajectory of the electrons may be described in terms of a transport matrix. In Appendix F, we show that the transverse emittance

$$
\begin{aligned}
\varepsilon(\psi)= & \lambda_{0} \sin ^{-2}\left(\theta_{0} / 2\right) \\
& \times \sqrt{\left\langle\delta C_{i}^{2}(\psi)\right\rangle\left\langle\delta \dot{C}_{i}^{2}(\psi)\right\rangle-\left\langle\delta C_{i}(\psi) \delta \dot{C}_{i}(\psi)\right\rangle^{2}}
\end{aligned}
$$

is conserved along the interaction region

$$
\varepsilon(\psi=\infty)=\varepsilon(\psi=0) .
$$

This is the fourth important result characteristic to this guidance paradigm.

\section{Envelope equation}

Knowing for a pencil beam that the emittance is conserved, we can go one step further and develop the envelope equation for the OBB. Let us consider a particle at the vacuum-beam interface-its radius is denoted by $R_{b}$. Ignoring space charge and angular momentum, according to Eq. (15) the particle's trajectory is oscillating

$$
\frac{d^{2} R_{b}}{d s^{2}}+\left(\frac{e E_{0}}{2^{3 / 2} m c^{2} \bar{\gamma}}\right)^{2} R_{b}=0,
$$

where $s=c t$. For what follows, it is convenient to define $K \equiv e E_{0} / 2^{3 / 2} m c^{2} \bar{\gamma}$, which represents the "lattice" periodicity.

Including the repelling space-charge force developed in Appendix D and in a similar way we incorporate the angular momentum term from Eq. (9), we obtain

$\frac{d^{2} R_{b}}{d s^{2}}+\frac{1}{8}\left(\frac{e E_{0}}{m c^{2} \bar{\gamma}}\right)^{2} R_{b}-\left(\frac{2 N_{\Delta} r_{\mathrm{el}}}{\bar{\gamma}^{3}}\right) \frac{1}{R_{b}}-\frac{\varepsilon^{2}}{R_{b}^{3}}=0$,

where $\varepsilon$ is the transverse emittance being related to the angular momentum by $\varepsilon=\mathcal{L} \lambda_{0} / \bar{\gamma}$. In this process, we ignored the energy spread by using, instead of $\gamma_{i}$, its average value $\bar{\gamma}=\left\langle\gamma_{i}\right\rangle ; r_{\mathrm{el}}$ is the classical radius of the electron.

The equation above represents the KapchinskijVladimirskij equation for the OBB. As such, it is evident that the beam's envelope has an equilibrium value that depends on the laser intensity, $e$-beam emittance, and number of electrons per unit length $N_{\Delta}$. In fact, it is convenient to define the "space-charge emittance" $\varepsilon_{\mathrm{sc}}=$ $N_{\Delta} r_{\mathrm{el}} / \bar{\gamma}^{3} \mathrm{~K}$ allowing us to write the equilibrium condition as 


$$
R_{b, \mathrm{eq}}^{2}=\frac{1}{K}\left[\varepsilon_{\mathrm{sc}}+\sqrt{\varepsilon_{\mathrm{sc}}^{2}+\varepsilon^{2}}\right] \simeq \begin{cases}2 \varepsilon_{\mathrm{sc}} / K & \varepsilon_{\mathrm{sc}} \gg \varepsilon, \\ \varepsilon / K & \varepsilon_{\mathrm{sc}} \ll \varepsilon,\end{cases}
$$

which indicates that, if the equilibrium is space-charge dominated $\left(\varepsilon_{\mathrm{sc}} \gg \varepsilon\right)$, the beam radius is independent of the emittance, whereas, in the opposite case, $R_{b \text {, } \mathrm{q}}^{2} \propto \varepsilon$.

\section{CONCLUSIONS}

In conclusion, we considered the trajectories of electrons counterpropagating along a radially polarized optical Bessel beam. It was shown that a significant fraction of the electrons can be transversally trapped by the OBB even in the case of "unmatched" injection, i.e., an arbitrary phase with respect to the optical field. Moreover, these transversally trapped particles can be transported without loss over more than $10^{5}$ laser wavelengths $(1 \mu \mathrm{m})$. The distance is limited only by the length of the OBB as long as there is full longitudinal overlap between the electrons and laser pulse. The unique profile $\left[J_{1}(u)\right]$ of the transverse field components supports the propagation of annular beams in addition to an azimuthally symmetric pencil beam in the vicinity of the zeros of the Bessel function, namely, $J_{1}(u)=0$. Qualitatively, space charge tends to suppress annular beams, and it reduces the amount of charge trapped on axis.

Assessment of the emittance of the TTPs alone reveals (see Table II) typical values of $10-50 \mathrm{pm}$ for the parameters specified in Table I. In fact, our simulations indicate that, if we trace the emittance of those particles that are eventually trapped from input to output, we find, within an error $\left(\propto N_{\mathrm{mp}}^{-1 / 2}\right)$ associated with a relatively small number of microparticles (70-40), that this emittance is conserved.

To thoroughly investigate the processes involved, we developed an analytic model whereby we averaged over the fast oscillation associated with the counterpropagating electron and Bessel laser beam. The resulting Hamiltonian has a Bessel (radial) potential $J_{1}^{2}(u)$ which, when operated in the linear regime and near equilibrium, causes rotation of the phase space, and using this we determined the analytic condition $\left(e E_{0} \lambda_{0} / m c^{2} \bar{\gamma}\right)^{2}=$ $2\left\langle\dot{x}_{i}^{2}\right\rangle\left\langle x_{i}^{2}\right\rangle^{-1}$ for the emittance to be conserved.

Finally, our choice of counterpropagating electrons is not arbitrary. In Appendix G, we show that, while the stability is identical in both cases, namely, the oscillation follows the same frequency, the transverse confinement (kick) is $\left(\theta_{0} / 2\right)^{2}$ weaker in the copropagating case.

\section{ACKNOWLEDGMENTS}

This study was supported by the Israel Science Foundation (ISF) and STI Optronics, Inc. Internal Research and Development.

\section{APPENDIX A: EQUATIONS OF MOTION}

From the explicit expressions of the potentials [Eq. (1)] that satisfy the Lorentz gauge, we may derive the three nonzero field components in the cylindrical coordinates system:

$$
\begin{aligned}
\mu_{0} H_{\phi} & =-\frac{\partial}{\partial r} A_{z}^{(\mathrm{OBB})}=-\frac{E_{0}}{c \sin \theta_{0}} J_{1}\left(\frac{\omega_{0}}{c} r \sin \theta_{0}\right) \sin \left[\omega_{0}\left(t+\frac{z}{c} \cos \theta_{0}\right)\right], \\
E_{r} & =-\frac{\partial}{\partial r} \Phi^{(\mathrm{OBB})}=\frac{E_{0} \cos \theta_{0}}{\sin \theta_{0}} J_{1}\left(\frac{\omega_{0}}{c} r \sin \theta_{0}\right) \sin \left[\omega_{0}\left(t+\frac{z}{c} \cos \theta_{0}\right)\right], \\
E_{z} & =-\frac{\partial}{\partial t} A_{z}^{(\mathrm{OBB})}-\frac{\partial}{\partial z} \Phi^{(\mathrm{OBB})}=E_{0} J_{0}\left(\frac{\omega_{0}}{c} r \sin \theta_{0}\right) \cos \left[\omega_{0}\left(t+\frac{z}{c} \cos \theta_{0}\right)\right] .
\end{aligned}
$$

With these field components, we may write the equation of motion of the $i$ th electron in the Cartesian coordinate system:

$$
\begin{aligned}
\frac{d}{d t}\left(\gamma_{i} \beta_{x, i}\right) & =-\frac{e}{m c}\left(E_{x, i}-c \mu_{0} \beta_{z, i} H_{y, i}\right), \\
\frac{d}{d t}\left(\gamma_{i} \beta_{y, i}\right) & =-\frac{e}{m c}\left(E_{y, i}+c \mu_{0} \beta_{z, i} H_{x, i}\right), \\
\frac{d}{d t}\left(\gamma_{i} \beta_{z, i}\right) & =-\frac{e}{m c}\left(E_{z, i}+c \mu_{0} \beta_{x, i} H_{y, i}-c \mu_{0} \beta_{y, i} H_{x, i}\right), \\
\frac{d \gamma_{i}}{d t} & =-\frac{e}{m c^{2}}\left(c \beta_{x, i} E_{x, i}+c \beta_{y, i} E_{y, i}+c \beta_{z} E_{z}\right), \\
\chi_{i} & =\omega_{0}\left(t+\frac{z_{i}}{c} \cos \theta_{0}\right) .
\end{aligned}
$$


The last expression determines the phase experienced by the $i$ th particle. Keeping in mind that in cylindrical coordinates $E_{x}=E_{r} \cos \phi$ and $x=r \cos \phi$, we get $E_{x}=(x / r) E_{r}$. Similarly, $E_{y}=(y / r) E_{r}, H_{x}=-H_{\phi} \sin \phi=$ $-(y / r) H_{\phi}$, and $H_{y}=H_{\phi} \cos \phi=(x / r) H_{\phi}$. Thus, we have

$$
\begin{aligned}
\frac{d}{d t}\left(\gamma_{i} \beta_{x, i}\right) & =-\frac{e}{m c}\left(E_{r, i}-\eta_{0} \beta_{z, i} H_{\phi, i}\right) \frac{x_{i}}{r_{i}} \\
\frac{d}{d t}\left(\gamma_{i} \beta_{y, i}\right) & =-\frac{e}{m c}\left(E_{r, i}-\eta_{0} \beta_{z, i} H_{\phi, i}\right) \frac{y_{i}}{r_{i}} \\
\frac{d}{d t}\left(\gamma_{i} \beta_{z, i}\right) & =-\frac{e}{m c}\left(E_{z, i}+\eta_{0} \beta_{x, i} \frac{x_{i}}{r_{i}} H_{\phi, i}+\eta_{0} \beta_{y, i} \frac{y_{i}}{r_{i}} H_{\phi, i}\right), \\
\frac{d}{d t} \gamma_{i} & =-\frac{e}{m c}\left(\beta_{x, i} \frac{x_{i}}{r_{i}} E_{r, i}+\beta_{y, i} \frac{y_{i}}{r_{i}} E_{r, i}+\beta_{z} E_{z}\right), \\
\frac{d}{d t} \chi_{i} & =\omega_{0}\left(1+\beta_{z, i} \cos \theta_{0}\right) .
\end{aligned}
$$

At this stage, we may substitute the explicit expressions for the field components derived in Eq. (A2), and, defining $\psi_{i}=\frac{\omega_{0}}{c} r_{i} \sin \theta_{0}$ as the argument of the Bessel functions, we find

$$
\begin{aligned}
\frac{d}{d t}\left(\gamma_{i} \beta_{x, i}\right) & =-\left[\frac{e}{m c}\left(\cos \theta_{0}+\beta_{z, i}\right) \frac{E_{0}}{\sin \theta_{0}} J_{1}\left(\psi_{i}\right) \sin \chi_{i}\right] \frac{x_{i}}{r_{i}}, \\
\frac{d}{d t}\left(\gamma_{i} \beta_{y, i}\right) & =-\left[\frac{e}{m c}\left(\cos \theta_{0}+\beta_{z, i}\right) \frac{E_{0}}{\sin \theta_{0}} J_{1}\left(\psi_{i}\right) \sin \chi_{i}\right] \frac{y_{i}}{r_{i}}, \\
\frac{d}{d t}\left(\gamma_{i} \beta_{z, i}\right) & =-\frac{e}{m c}\left[E_{0} J_{0}\left(\psi_{i}\right) \cos \chi_{i}-\left(\beta_{x, i} \frac{x_{i}}{r_{i}}+\beta_{y, i} \frac{y_{i}}{r_{i}}\right) \frac{E_{0}}{\sin \theta_{0}} J_{1}\left(\psi_{i}\right) \sin \chi_{i}\right], \\
\frac{d}{d t} \gamma_{i} & =-\frac{e}{m c}\left[\left(\beta_{x, i} \frac{x_{i}}{r_{i}}+\beta_{y, i} \frac{y_{i}}{r_{i}}\right) \frac{E_{0} \cos \theta_{0}}{\sin \theta_{0}} J_{1}\left(\psi_{i}\right) \sin \chi_{i}+\beta_{z, i} E_{0} J_{0}\left(\psi_{i}\right) \cos \chi_{i}\right], \\
\frac{d}{d t} \chi_{i} & =\omega_{0}\left(1+\beta_{z, i} \cos \theta_{0}\right) .
\end{aligned}
$$

Defining the normalized quantities as

$$
\begin{aligned}
\tau & =c t / \lambda_{0}, \quad \bar{x}_{i}=x_{i} / \lambda_{0}, \quad \bar{y}_{i}=y_{i} / \lambda_{0}, \quad \bar{z}_{i}=z_{i} / \lambda_{0}, \quad \psi_{i}=2 \pi \sqrt{\bar{x}_{i}^{2}+\bar{y}_{i}^{2}} \sin \theta_{0}, \\
\bar{U}_{i} & =\pi \bar{E} J_{c}\left(\psi_{i}\right) \sin \left(\chi_{i}\right), \quad \bar{V}_{i}=\bar{E} J_{0}\left(\psi_{i}\right) \cos \left(\chi_{i}\right), \quad \bar{E}=e E_{0} \lambda_{0} / m c^{2}, \quad J_{c}(u) \equiv 2 J_{1}(u) / u,
\end{aligned}
$$

we finally get

$$
\begin{aligned}
\frac{d}{d \tau}\left(\gamma_{i} \dot{\bar{x}}_{i}\right) & =-\left(\cos \theta_{0}+\dot{\bar{z}}_{i}\right) \bar{x}_{i} \bar{U}_{i}, \\
\frac{d}{d \tau}\left(\gamma_{i} \dot{\bar{y}}_{i}\right) & =-\left(\cos \theta_{0}+\dot{\bar{z}}_{i}\right) \bar{y}_{i} \bar{U}_{i}, \\
\frac{d}{d \tau}\left(\gamma_{i} \dot{\bar{z}}_{i}\right) & =\left[\frac{1}{2} \frac{d}{d \tau}\left(\bar{x}_{i}^{2}+\bar{y}_{i}^{2}\right)\right] \bar{U}_{i}-\bar{V}_{i}, \\
\frac{d \gamma_{i}}{d \tau} & =-\cos \theta_{0}\left[\frac{1}{2} \frac{d}{d \tau}\left(\bar{x}_{i}^{2}+\bar{y}_{i}^{2}\right)\right] \bar{U}_{i}-\dot{\bar{z}}_{i} \bar{V}_{i}, \\
\frac{d \chi_{i}}{d \tau} & =2 \pi\left(1+\dot{\bar{z}}_{i} \cos \theta_{0}\right),
\end{aligned}
$$

which constitutes the fundamental set of equations introduced in Eq. (2) and used throughout this study.

\section{APPENDIX B: CANONICAL LONGITUDINAL} MOMENTUM AND ENERGY SPREAD

The conservation of the longitudinal canonical momentum [Eq. (6)] implies that for relativistic particles, $\gamma_{i}$ (and $\left.\chi_{i}\right)$ can be solved analytically:

$$
\begin{aligned}
\gamma_{i}(\tau) & +\frac{\bar{E} \sin \left[\chi_{i}(0)+2 \pi\left(1+\cos \theta_{0}\right) \tau\right]}{2 \pi\left(1+\cos \theta_{0}\right)} \\
= & \gamma_{i}(0)+\frac{\bar{E} \sin \left[\chi_{i}(0)\right]}{2 \pi\left(1+\cos \theta_{0}\right)} .
\end{aligned}
$$

From this last expression, we may conclude that, if the initial energy and phase are statistically independent, the average energy is independent of the OBB, i.e., $\left\langle\gamma_{i}(\tau)\right\rangle=$ $\left\langle\gamma_{i}(0)\right\rangle$, whereas the second moment is given by 


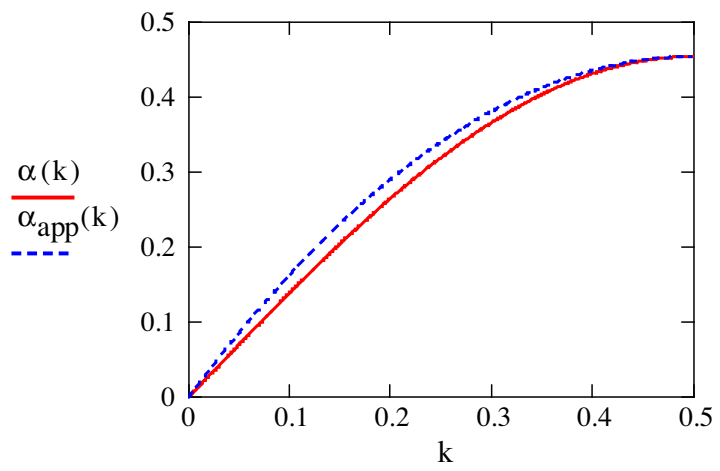

(a)

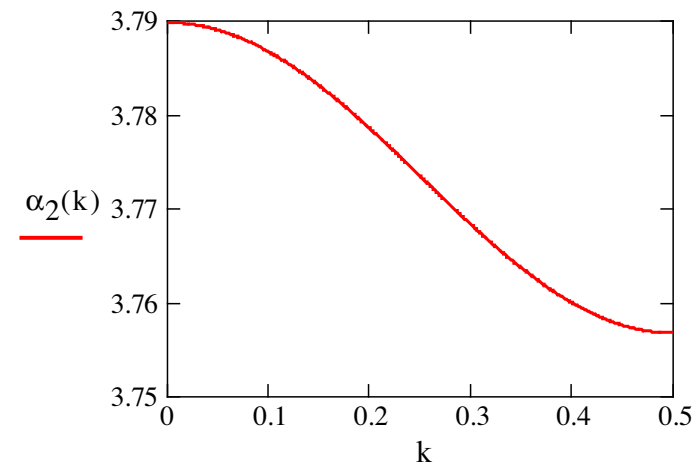

(b)

FIG. 5. Dispersion curves corresponding to solution of Eq. (C2). (a) In red is the first passband and in blue its polynomial approximation. (b) The second passband is relatively far away from the first one, and it is one order of magnitude narrower. The eigenvalues are independent of the angle $\chi_{0}$.

$$
\begin{aligned}
\left\langle\gamma_{i}^{2}(\tau)\right\rangle_{i}= & \left\langle\gamma_{i}^{2}(0)\right\rangle_{i}+\left[\frac{\bar{E}}{2 \pi\left(1+\cos \theta_{0}\right)}\right]^{2} \\
& \times\left\{1-\cos \left[2 \pi\left(1+\cos \theta_{0}\right) \tau\right]\right\} .
\end{aligned}
$$

Averaging in time over one oscillation period, we conclude that the initial energy spread $\Delta \gamma_{\text {ini }}^{2}$ increases according to

$$
\Delta \gamma^{2}=\Delta \gamma_{\mathrm{ini}}^{2}+\left[\frac{e E_{0} \lambda_{0} / m c^{2}}{2 \pi\left(1+\cos \theta_{0}\right)}\right]^{2}
$$

This last result represents the electrons' energy spread increase when the beam enters the OBB region. Because of conservation of longitudinal canonical momentum, once the $e$-beam exits the $\mathrm{OBB}$, the electrons' energy spread drops back to its original value.

\section{APPENDIX C: MATHIEU'S EQUATION}

In this Appendix, we determine the constraints on $\alpha$ that ensure a periodic (stable) solution to Mathieu's equation

$$
\left[\frac{d^{2}}{d u^{2}}+\alpha \sin \left(u+\chi_{0}\right)\right] \Psi(u)=0,
$$

namely, $\Psi(u)=\exp (-j k u) \sum_{n=-\infty}^{\infty} \bar{\Psi}_{n} \exp (-j n u)$, where $u=(-\pi, \pi)$ and $|k|<0.5$ is the projection on the first Brillouin zone. The Mathieu equation now has an algebraic form

$$
\sum_{n, n^{\prime}=-\infty}^{\infty}\left[M_{n, n^{\prime}}-\alpha^{-1} \delta_{n, n^{\prime}}\right] \tilde{\Psi}_{n^{\prime}} \exp (-j n u)=0,
$$

with $\quad M_{n, n^{\prime}} \equiv \frac{1}{2 j}\left(k+n^{\prime}\right)^{-2}\left[\delta_{n+1, n^{\prime}} \exp \left(-j \chi_{0}\right)-\delta_{n-1, n^{\prime}} \times\right.$ $\left.\exp \left(+j \chi_{0}\right)\right], \tilde{\Psi}_{n^{\prime}} \equiv\left(k+n^{\prime}\right)^{2} \bar{\Psi}_{n^{\prime}}$. Equation (C1) is an eigenvalue problem, or, explicitly, $\alpha^{-1}$ represents the eigenvalues of the matrix $M$. In the framework of a periodic solution, it can be shown that the eigenvalues are independent of the initial angle $\chi_{0}$, and within a reasonable approximation the first passband in the first Brillouin zone can be approximated by $\alpha(k) \simeq 4 \alpha_{\max }\left|k-k^{2}\right|, \quad$ where $\quad 0 \leq \alpha(k) \leq \alpha_{\max } \equiv \alpha(k=$ $0.5) \simeq 0.454$. The red curve in Fig. 5(a) shows the numerical solution for $|n| \leq 5$, whereas in blue we plot the approximation from the above. After a wide forbidden gap (corresponding to unconfined trajectories), the second branch $\alpha_{2}(k)$ is one order of magnitude narrower, i.e., $\alpha_{\max }=3.79, \alpha_{\min }=3.757$. See Fig. 5(b).

\section{APPENDIX D: SPACE CHARGE}

In free space, an azimuthally symmetric pencil beam of uniform density tends to diverge due to the net repelling force $F_{r}^{(\mathrm{sc})}=r e^{2}\left(N / \pi R_{b}^{2} \Delta_{z}\right) /\left(2 \gamma^{2} \varepsilon_{0}\right)$ within the volume of the beam. $N$ is the number of electrons in the volume $\pi R_{b}^{2} \Delta_{z}$, and the beam radius is $R_{b}$. Actually, as we show subsequently, the quantity that matters is the number of electrons $N$ per unit length $\Delta_{z}$; thus, we define $N_{\Delta} \equiv N / \Delta_{z}$. In practice, the radial distribution varies in time, and, therefore, the space-charge force (after averaging over the longitudinal dimension) is

$$
F_{r}^{(\mathrm{sc})}(r, t)=\frac{e^{2} N_{\Delta}}{2 \pi \varepsilon_{0}} \frac{1}{\gamma^{2}} \frac{1}{r}\left\langle h\left[r-r_{i}(t)\right]\right\rangle_{i} .
$$

The earlier expression for the radial force is readily retrieved if we notice that for a uniform distribution $\left(2 / R_{b}^{2}\right) \int_{0}^{R_{b}} d \rho \rho h(r-\rho)=r^{2} / R_{b}^{2}$ if $r \leq R_{b}$ and 1 otherwise. Consequently, by analogy to the latter and the fact that for a uniform distribution $\left\langle r^{2}\right\rangle_{u}=\frac{2}{R_{b}^{2}} \int_{0}^{R_{b}} d \rho \rho^{3}=$ $\frac{1}{2} R_{b}^{2}$, we consider the following approximation for the space-charge term:

$$
\left\langle h\left[r-r_{i}(t)\right]\right\rangle_{i} \simeq \begin{cases}\frac{r^{2}}{2\left\langle r_{i}^{2}\right\rangle_{i}} & r^{2} \leq 2\left\langle r_{i}^{2}\right\rangle_{i}, \\ 1 & r^{2}>2\left\langle r_{i}^{2}\right\rangle_{i}\end{cases}
$$


This is obviously an approximation that becomes exact for the case of a uniform radial distribution, but it obviously fails for large deviations. Therefore, we can expect it to describe properly a pencil-beam-like configuration but be less accurate when an annular beam is involved. In the latter case, at best this model provides us with a general trend. Despite these limitations, this approach greatly speeds up the numerical processing time while still providing rough assessments.

Subject to the assumptions above, the transverse spacecharge forces can be readily incorporated in the equations of motion [Eq. (2)], which then read

$$
\begin{aligned}
\frac{d}{d \tau}\left(\gamma_{i} \dot{\bar{x}}_{i}\right) & =-\left[\left(\cos \theta_{0}+\dot{\bar{z}}_{i}\right) \bar{U}_{i}-h_{i} \frac{1}{\gamma_{i}^{2}} \frac{1}{\bar{r}_{i}^{2}}\right] \bar{x}_{i}, \\
\frac{d}{d \tau}\left(\gamma_{i} \dot{\bar{y}}_{i}\right) & =-\left[\left(\cos \theta_{0}+\dot{\bar{z}}_{i}\right) \bar{U}_{i}-h_{i} \frac{1}{\gamma_{i}^{2}} \frac{1}{\bar{r}_{i}^{2}}\right] \bar{y}_{i}, \\
\frac{d}{d \tau}\left(\gamma_{i} \dot{\bar{z}}_{i}\right) & =\left(\frac{1}{2} \frac{d \bar{r}_{i}^{2}}{d \tau}\right)\left(\bar{U}_{i}+\frac{1}{\bar{r}_{i}^{2}} h_{i} \dot{\bar{z}}_{i}\right)-\bar{V}_{i}, \\
\frac{d \gamma_{i}}{d \tau} & =-\left(\frac{1}{2} \frac{d \bar{r}_{i}^{2}}{d \tau}\right)\left(\cos \theta_{0} \bar{U}_{i}-h_{i} \frac{1}{\bar{r}_{i}^{2}}\right)-\dot{\bar{z}}_{i} \bar{V}_{i}, \\
\frac{d \chi_{i}}{d \tau} & =2 \pi\left(1+\dot{\bar{z}}_{i} \cos \theta_{0}\right), \\
h_{i} & =\underbrace{2 r_{\mathrm{el}} N_{\Delta}}_{\equiv n_{\mathrm{sc}}}\left\{\begin{array}{cc}
\frac{\bar{r}_{i}^{2}}{\overline{\bar{r}}_{b}^{2}} & \bar{r}_{i}^{2} \leq \bar{r}_{b}^{2}, \\
1 & \bar{r}_{i}^{2}>\bar{r}_{b}^{2},
\end{array}\right.
\end{aligned}
$$

wherein $\bar{r}_{b}^{2}=2\left\langle\bar{r}_{i}^{2}\right\rangle$, and $r_{\mathrm{el}}=2.8 \times 10^{-15}[\mathrm{~m}]$ is the classical radius of the electron. For an assessment of the value of the space-charge parameter, let us consider first an example density of $10^{9}$ electrons confined by a $(100 \mu \mathrm{m})^{3}$ cube; thus, $n_{b} \simeq 10^{21}\left[\mathrm{~m}^{-3}\right]$. A beam of similar density but radius $R_{b}=5[\mu \mathrm{m}]$ contains $N_{\Delta} \simeq 0.75 \times 10^{11}\left[\mathrm{~m}^{-1}\right]$; thus, $\kappa_{\mathrm{sc}} \equiv 2 r_{\mathrm{el}} N_{\Delta} \simeq 4.2 \times 10^{-4}$. For stability, we require that the transverse force exerted by the $\mathrm{OBB}$ is dominant: $\left|\left(\cos \theta_{0}+\dot{\bar{z}}_{i}\right) \bar{U}_{i}\right|_{\max } \gg \kappa_{\mathrm{sc}} \gamma_{i}^{-2} \Rightarrow 2 \pi e E_{0} \lambda_{0} / m c^{2} \gg 2 r_{\mathrm{el}} N_{\Delta} \gamma_{i}^{-2}$. For the parameters specified above, this is readily satisfied.

\section{APPENDIX E: EFFECTIVE HAMILTONIAN}

Our purpose is to develop an analytic approach to simplify the equation of motion taking advantage of the fact that the electron and the laser are counterpropagating; thus, the absolute phase varies rapidly. We further neglect the energy variations and consider zero angular momentum in Eq. (9); thus,

$$
\frac{d^{2} \rho_{i}}{d \psi^{2}} \simeq-\alpha_{i} J_{1}\left(\rho_{i}\right) \sin \left(\chi_{i, 0}+\psi\right)
$$

wherein $\quad \psi=2 \pi \tau\left(1+\cos \theta_{0}\right), \quad \rho_{i}=2 \pi \bar{r}_{i} \sin \theta_{0}, \quad$ and $\alpha_{i}=\bar{E}\left[2 \pi \gamma_{i, 0}\left(1+\cos \theta_{0}\right)\right]^{-1}$. The radial location of the electron has a slowly varying component and a fast varying one:

$$
\begin{aligned}
& \rho_{i}=A_{i} \cos \left(\chi_{i, 0}+\psi\right)+B_{i} \sin \left(\chi_{i, 0}+\psi\right)+C_{i}, \\
& \dot{\rho}_{i} \equiv \frac{d \rho_{i}}{d \psi}=\left(\dot{A}_{i}+B_{i}\right) \cos \left(\chi_{i, 0}+\psi\right)+\left(\dot{B}_{i}-A_{i}\right) \sin \left(\chi_{i, 0}+\psi\right)+\dot{C}_{i}, \\
& \ddot{\rho}_{i}=\left(\ddot{A}_{i}-A_{i}+2 \dot{B}_{i}\right) \cos \left(\chi_{i, 0}+\psi\right)+\left(\ddot{B}_{i}-2 \dot{A}_{i}-B_{i}\right) \sin \left(\chi_{i, 0}+\psi\right)+\ddot{C}_{i},
\end{aligned}
$$

for substituting in Eq. (E2), we present also the first and second derivative of $\rho_{i}$ :

$$
\begin{aligned}
& \left(\ddot{A}_{i}-A_{i}+2 \dot{B}_{i}\right) \cos \left(\chi_{i, 0}+\psi\right)+\left(\ddot{B}_{i}-2 \dot{A}_{i}-B_{i}\right) \sin \left(\chi_{i, 0}+\psi\right)+\ddot{C}_{i} \\
& \simeq-\alpha_{i} J_{1}\left[A_{i} \cos \left(\chi_{i, 0}+\psi\right)+B_{i} \sin \left(\chi_{i, 0}+\psi\right)+C_{i}\right] \sin \left(\chi_{i, 0}+\psi\right),
\end{aligned}
$$

and using the orthogonality of the trigonometric functions we get

$$
\begin{aligned}
\ddot{C}_{i} & \simeq-\alpha_{i} \frac{1}{2 \pi} \int_{0}^{2 \pi} d \psi J_{1}\left[A_{i} \cos (\psi)+B_{i} \sin (\psi)+C_{i}\right] \sin (\psi), \\
\ddot{A}_{i}-A_{i}+2 \dot{B}_{i} & \simeq-\alpha_{i} \frac{1}{2 \pi} \int_{0}^{2 \pi} d \psi J_{1}\left[A_{i} \cos (\psi)+B_{i} \sin (\psi)+C_{i}\right] \sin (2 \psi), \\
\ddot{B}_{i}-2 \dot{A}_{i}-B_{i} & \simeq-2 \alpha_{i} \frac{1}{2 \pi} \int_{0}^{2 \pi} d \psi J_{1}\left[A_{i} \cos (\psi)+B_{i} \sin (\psi)+C_{i}\right] \sin ^{2}(\psi) .
\end{aligned}
$$

In the framework of the slowly varying amplitude approximation, the derivatives of $A$ and $B$ are assumed to be negligible. Furthermore, we keep in mind that, by defining $a_{i}=\sqrt{A_{i}^{2}+B_{i}^{2}}, \theta_{i} \equiv \arctan \left(B_{i} / A_{i}\right)$, the argument of the Bessel function reads $C_{i}+a_{i} \cos \left(\psi-\theta_{i}\right)$; therefore, we get 


$$
\begin{aligned}
& \ddot{C}_{i} \simeq-\alpha_{i} \frac{1}{2 \pi} \int_{0}^{2 \pi} d \psi J_{1}\left[C_{i}+a_{i} \cos (\psi)\right] \sin \left(\psi+\theta_{i}\right) \simeq-\alpha_{i} \frac{1}{2}\left[\frac{d J_{1}(\chi)}{d \chi}\right]_{\chi=C_{i}} B_{i}, \\
& A_{i} \simeq 2 \alpha_{i} \frac{1}{2 \pi} \int_{0}^{2 \pi} d \psi J_{1}\left[C_{i}+a_{i} \cos (\psi)\right] \frac{1}{2} \sin \left(2 \psi+2 \theta_{i}\right) \simeq O\left(a^{2}\right), \\
& B_{i} \simeq 2 \alpha_{i} \frac{1}{2 \pi} \int_{0}^{2 \pi} d \psi J_{1}\left[C_{i}+a_{i} \cos (\psi)\right] \frac{1}{2}\left[1-\cos \left(2 \psi+2 \theta_{i}\right)\right] \simeq \alpha_{i} J_{1}\left(C_{i}\right)+O\left(a^{2}\right),
\end{aligned}
$$

where we again employed the slowly varying amplitude approximation; namely, we assume that the amplitudes and the phase vary slowly compared to trigonometric functions. Based on the last three expressions, we have $A_{i} \simeq 0$, $B_{i} \simeq \alpha_{i} J_{1}\left(C_{i}\right)$, and

$$
\frac{d^{2}}{d \psi^{2}} C_{i} \simeq-\frac{1}{4} \alpha_{i}^{2} \frac{d}{d C_{i}} J_{1}^{2}\left(C_{i}\right) .
$$

So we need to solve only the latter, since once $C_{i}$ is known, the radial coordinate is known: $\rho_{i}=\alpha_{i} J_{1}\left(C_{i}\right) \times$ $\sin \left(\chi_{i, 0}+\psi\right)+C_{i}$, wherein again the first term is the fast oscillatory term of the radial location, whereas the second is its slowly varying counterpart.

Based on this equation of motion, we can deduce the Hamiltonian associated with the radial motion, as we ignored the change in the total kinetic energy. For this purpose, we multiply the equation of motion by $d C_{i} / d \psi$ :

$$
\begin{aligned}
& \frac{d}{d \psi}\left[\frac{1}{2}\left(\frac{d C_{i}}{d \psi}\right)^{2}+\frac{1}{4} \alpha_{i}^{2} J_{1}^{2}\left(C_{i}\right)\right] \\
& =0 \Rightarrow \mathcal{H}=\frac{1}{2}\left(\frac{d C_{i}}{d \psi}\right)^{2}+\frac{1}{4} \alpha_{i}^{2} J_{1}^{2}\left(C_{i}\right),
\end{aligned}
$$

wherein $\mathcal{H}$ is the normalized Hamiltonian and obviously it is positively defined, i.e., $\mathcal{H} \geq 0$.

\section{APPENDIX F: EMITTANCE OF THE TRANSPORTED BEAM}

In this Appendix, we examine the emittance as electrons are oscillating near one of the zeros of the potential $p_{1, s}$ : $J_{1}\left(p_{1, s}\right) \equiv 0, s=0,1,2, \ldots$. Consider a linearized solution of the form $C_{i} \simeq p_{1, s}+\delta C_{s, i}$, and then we may write

$$
\begin{aligned}
& \frac{d}{d \bar{\psi}} {\left[\frac{1}{2}\left(\frac{d \delta C_{s, i}}{d \psi}\right)^{2}+\frac{1}{4} \alpha_{i}^{2} J_{0}^{2}\left(p_{1, s}\right)\left(\delta C_{s, i}\right)^{2}\right] } \\
&=0 \Rightarrow\left[\frac{d^{2}}{d \psi^{2}}+\frac{1}{2} \alpha_{i}^{2} J_{0}^{2}\left(p_{1, s}\right)\right] \delta C_{s, i}=0 .
\end{aligned}
$$

Defining the normalized oscillating frequency $\omega_{s, i}^{2} \equiv$ $(1 / 2) \alpha_{i}^{2} J_{0}^{2}\left(p_{1, s}\right) \equiv \bar{\omega}_{s}^{2} \bar{\gamma}^{2} \gamma_{i}^{-2}$ and keeping in mind that, for most practical acceleration applications, the energy spread is of the order $\sim 0.1 \%$, we can assume $\bar{\gamma} \gamma_{i}^{-1} \sim 1$, wherein $\bar{\gamma}=\left\langle\gamma_{i}\right\rangle$. As a result, the transport matrix that describes the trajectory is

$$
\left[\begin{array}{l}
\delta C_{s, i}(\psi) \\
\delta \dot{C}_{s, i}(\psi)
\end{array}\right]=\left[\begin{array}{cc}
\cos \left(\bar{\omega}_{s} \psi\right) & \frac{\sin \left(\bar{\omega}_{s} \psi\right)}{\bar{\omega}_{s}} \\
-\bar{\omega}_{s} \sin \left(\bar{\omega}_{s} \psi\right) & \cos \left(\bar{\omega}_{s} \psi\right)
\end{array}\right]\left[\begin{array}{l}
\delta C_{s, i}(0) \\
\delta \dot{C}_{s, i}(0)
\end{array}\right],
$$

that has a determinant of unity corresponding to a rotation of the phase space. In the framework of the present notation, the emittance is given by

$$
\begin{aligned}
\varepsilon(\psi)= & \lambda_{0} \sin ^{-2}\left(\theta_{0} / 2\right) \\
& \times \sqrt{\left\langle\delta C_{i}^{2}(\psi)\right\rangle\left\langle\delta \dot{C}_{i}^{2}(\psi)\right\rangle-\left\langle\delta C_{i}(\psi) \delta \dot{C}_{i}(\psi)\right\rangle^{2}} .
\end{aligned}
$$

Consequently, after the transients have decayed, $\bar{\omega}_{s} \psi \gg \pi$, the emittance is given by

$$
\begin{aligned}
\varepsilon_{\infty} & \equiv \varepsilon(\psi \rightarrow \infty)=\lambda_{0} \sin ^{-2}\left(\theta_{0} / 2\right) \sqrt{\left\langle\overline{\left\langle C_{s, i}^{2}\right.}\right\rangle_{\infty}\left\langle\overline{\delta \dot{C}_{s, i}^{2}}\right\rangle_{\infty}} \\
& \simeq \frac{1}{2} \lambda_{0} \sin ^{-2}\left(\theta_{0} / 2\right)\left[\bar{\omega}_{s}\left\langle\delta C_{s, i}^{2}(0)\right\rangle+\frac{1}{\bar{\omega}_{s}}\left\langle\delta \dot{C}_{s, i}^{2}(0)\right\rangle\right],
\end{aligned}
$$

where we define $\overline{F^{2}(u)} \equiv \frac{1}{2 \pi} \int_{u-\pi}^{u+\pi} d x F^{2}(x)$. The best case scenario $d \varepsilon_{\infty} / d \bar{\omega}_{s}=0$ is achieved by matching the potential, represented by $\bar{\omega}_{s}$, to the initial phase space:

$$
\begin{aligned}
\bar{\omega}_{s} & =\sqrt{\left\langle\delta \dot{C}_{s, i}^{2}(0)\right\rangle\left\langle\delta C_{s, i}^{2}(0)\right\rangle^{-1}} \Rightarrow \frac{e E_{0} \lambda_{0}}{m c^{2} \bar{\gamma}} \\
& =\frac{\sqrt{2}}{\left|J_{0}\left(p_{1, s}\right)\right|} \sqrt{\left\langle(d \delta x / d \tau)_{s, i}^{2}\right\rangle_{\tau=0}\left\langle\delta x_{s, i}^{2}\right\rangle_{\tau=0}^{-1}} .
\end{aligned}
$$

Subject to this condition, the emittance is conserved along the entire interaction length:

$$
\varepsilon_{\infty}=\varepsilon(\psi=0)
$$

\section{APPENDIX G: CO- AND COUNTERPROPAGATING BEAMS}

The goal in Appendix G is to compare the dynamics of co- and counterpropagating beams. To obtain the equations that describe the copropagating motion, we switch the trajectory of the particles in the $z$ direction: $z_{i}(t) \rightarrow-z_{i}(t)$. Therefore, instead of Eq. (2), the system is described by 


$$
\begin{aligned}
\frac{d}{d \tau}\left(\gamma_{i} \dot{\bar{x}}_{i}\right) & =-\left(\cos \theta_{0}-\dot{\bar{z}}_{i}\right) \bar{x}_{i} \pi \bar{E} \sin \left(\chi_{i}\right), \\
\frac{d}{d \tau}\left(\gamma_{i} \dot{\bar{y}}_{i}\right) & =-\left(\cos \theta_{0}-\dot{\bar{z}}_{i}\right) \bar{y}_{i} \pi \bar{E} \sin \left(\chi_{i}\right), \\
\frac{d}{d \tau}\left(\gamma_{i} \dot{\bar{z}}_{i}\right) & =-\left[\frac{1}{2} \frac{d}{d \tau}\left(\bar{x}_{i}^{2}+\bar{y}_{i}^{2}\right)\right] \pi \bar{E} \sin \left(\chi_{i}\right)+\bar{E} \cos \left(\chi_{i}\right), \\
\frac{d \gamma_{i}}{d \tau} & =-\cos \theta_{0}\left[\frac{1}{2} \frac{d}{d \tau}\left(\bar{x}_{i}^{2}+\bar{y}_{i}^{2}\right)\right] \pi \bar{E} \sin \left(\chi_{i}\right)+\dot{\bar{z}}_{i} \bar{E} \cos \left(\chi_{i}\right), \\
\frac{d \chi_{i}}{d \tau} & =2 \pi\left(1-\dot{\bar{z}}_{i} \cos \theta_{0}\right),
\end{aligned}
$$

where the various normalizations are identical to those in the counterpropagating case. We focus on the transverse motion and ultrarelativistic case $\gamma \theta_{0} \gg 1$, and for comparison we present the corresponding equations for the counterpropagating case:

$$
\begin{array}{rlrl} 
& \text { Co - propagating } & \text { Counter - propagating } \\
\frac{d}{d \tau}\left(\gamma_{i} \dot{\bar{x}}_{i}\right) & =-\left(\cos \theta_{0}-\dot{\bar{z}}_{i}\right) \bar{x} \pi \bar{E} \sin \left(\chi_{i}\right) & \frac{d}{d \tau}\left(\gamma_{i} \dot{\bar{x}}_{i}\right) & =-\left(\cos \theta_{0}+\dot{\bar{z}}_{i}\right) \bar{x}_{i} \pi \bar{E} \sin \left(\chi_{i}\right) \\
& \simeq \frac{\pi}{2} \theta_{0}^{2} \bar{x}_{i} \bar{E} \sin \left(\chi_{i}\right) & & \simeq 2 \pi \bar{x}_{i} \bar{E} \sin \left(\chi_{i}\right) \\
\frac{d \chi_{i}}{d \tau} & =2 \pi\left(1-\dot{\bar{z}}_{i} \cos \theta_{0}\right) & \frac{d \chi_{i}}{d \tau} & =2 \pi\left(1+\dot{\bar{z}}_{i} \cos \theta_{0}\right) \\
& \simeq 2 \pi\left(\frac{1}{2 \gamma_{i}^{2}}+\frac{1}{2} \theta_{0}^{2}\right) \simeq \pi \theta_{0}^{2} & & \simeq 4 \pi
\end{array}
$$

It is evident that, for otherwise identical conditions, the confining force is $\left(\theta_{0} / 2\right)^{2}$ weaker in the copropagating case. This is the main reason for our choice of using a counterpropagating OBB.

Next, we focus our attention on the radial stability of the beam for both cases. We show for both cases that the slow varying component of the radial motion is stable. Subject to the previous assumptions, we may further assume that the electrons energy is constant; thus,

$$
\begin{array}{cc}
\text { Copropagating } & \text { Counter propagating } \\
\frac{d^{2} \bar{x}_{i}}{d u^{2}} \simeq \frac{1}{2 \pi \theta_{0}^{2}} \frac{\bar{E}}{\gamma} \sin u \bar{x}_{i}, & \frac{d^{2} \bar{x}_{i}}{d u^{2}} \simeq-\frac{1}{8 \pi} \frac{\bar{E}}{\gamma} \sin u \bar{x}_{i}, \\
u=\chi(0)+\pi \theta_{0}^{2} \tau, & u \simeq \chi(0)+4 \pi \tau .
\end{array}
$$

Note that the normalized time $(u)$ has a different (natural) definition. Clearly, the latter varies much slower in the copropagating case. In cylindrical coordinates, the motion may be explicitly written as

$$
\begin{aligned}
\bar{x}_{i} & =\rho_{i} \cos \phi_{i}, \\
\dot{\bar{x}}_{i} & \equiv \frac{d}{d u} \bar{x}_{i}=\dot{\rho}_{i} \cos \phi_{i}-\dot{\phi}_{i} \rho_{i} \sin \phi_{i}, \\
\ddot{\bar{x}}_{i} & =\ddot{\rho} \cos \phi_{i}-2 \dot{\phi}_{i} \dot{\rho}_{i} \sin \phi_{i}-\ddot{\phi}_{i} \rho_{i} \sin \phi_{i}-\dot{\phi}_{i}^{2} \rho_{i} \cos \phi_{i},
\end{aligned}
$$

and, assuming vanishing angular momentum (which is conserved), we get

\section{Copropagating Counterpropagating}

$$
\frac{d^{2} \rho_{i}}{d u^{2}} \simeq \frac{1}{2 \pi \theta_{0}^{2}} \frac{\bar{E}}{\gamma} \sin u \rho_{i}, \quad \frac{d^{2} \rho_{i}}{d u^{2}} \simeq-\frac{1}{8 \pi} \frac{\bar{E}}{\gamma} \sin u \rho_{i}
$$

The radial location of the electron has a fast varying component and a slowly varying one:

$$
\rho_{i}=A_{i} \cos u+B_{i} \sin u+C_{i},
$$


where the three unknown quantities $(A, B$, and $C)$ are slowly varying functions of $u$; thus,

$$
\begin{aligned}
& \dot{\rho}_{i} \equiv \frac{d}{d u} \rho_{i}=\left(\dot{A}_{i}+B_{i}\right) \cos u+\left(\dot{B}_{i}-A_{i}\right) \sin u+\dot{C}_{i}, \\
& \ddot{\rho}_{i}=\left(\ddot{A}_{i}-A_{i}+2 \dot{B}_{i}\right) \cos u+\left(\ddot{B}_{i}-2 \dot{A}_{i}-B_{i}\right) \sin u+\ddot{C}_{i} .
\end{aligned}
$$

Substituting in Eq. (G6) and using the orthogonality of $f_{0}(u)=1, f_{c}(u)=\cos u$, and $f_{s}(u)=\sin u$ for the copropagating case,

$$
\begin{aligned}
& f_{0}(u): \ddot{C}_{i}=\frac{\bar{E}}{4 \pi \theta_{0}^{2} \gamma_{i}} B_{i}, \\
& f_{c}(u):-A_{i}+2 \dot{B}_{i}=0 \Rightarrow A_{i} \simeq 0, \\
& f_{s}(u):-2 \dot{A}_{i}-B_{i} \simeq \frac{1}{2} \frac{\bar{E}}{\pi \theta_{0}^{2} \gamma_{i}} C_{i} \Rightarrow B_{i} \simeq-\frac{1}{2} \frac{\bar{E}}{\pi \theta_{0}^{2} \gamma_{i}} C_{i} .
\end{aligned}
$$

Combining the three results and repeating the procedure for the counterpropagating case, we obtain

$$
\begin{array}{rlrl}
\text { Co - propagating } & & \text { Counter - propagating } \\
A_{i} & \simeq 0 & A_{i} \simeq 0 \\
B_{i} \simeq-\frac{1}{2} \frac{\bar{E}}{\pi \theta_{0}^{2} \gamma_{i}} C_{i} & B_{i} \simeq \frac{1}{2} \frac{\bar{E}}{4 \pi \gamma_{i}} C_{i} \\
\frac{d^{2}}{d u^{2}} C_{i} \simeq-\frac{1}{8}\left(\frac{\bar{E}}{\pi \theta_{0}^{2} \gamma_{i}}\right)^{2} C_{i} & \frac{d^{2}}{d u^{2}} C_{i} \simeq-\frac{1}{8}\left(\frac{\bar{E}}{4 \pi \gamma_{i}}\right)^{2} C_{i} \\
u & =\chi(0)+\pi \theta_{0}^{2} \tau & & u=\chi(0)+4 \pi \tau
\end{array}
$$

Consequently, employing regular time normalization $\left(\tau=c t / \lambda_{0}\right)$ for both cases, we conclude that the radial motion of a narrow pencil beam is stable for both copropagating and counterpropagating beams, i.e.,

$$
\frac{d^{2}}{d \tau^{2}} C_{i} \simeq-\frac{1}{8}\left(\frac{\bar{E}}{\gamma_{i}}\right)^{2} C_{i}
$$

[1] B. Naranjo, A. Valloni, S. Putterman, and J. B. Rosenzweig, Stable Charged-Particle Acceleration and Focusing in a Laser Accelerator Using Spatial Harmonics, Phys. Rev. Lett. 109, 164803 (2012).

[2] U. Niedermayer, T. Egenolf, O. Boine-Frankenheim, and P. Hommelhoff, Alternating-Phase Focusing for Dielectric-Laser Acceleration, Phys. Rev. Lett. 121, 214801 (2018).

[3] M. Kozak, T. Eckstein, N. Schonenberger, and P. Hommelhoff, Inelastic ponderomotive scattering of electrons at a high-intensity optical travelling wave in vacuum, Nat. Phys. 14, 121 (2018).

[4] J. Arlt, V. Garces-Chavez, W. Sibbett, and K. Dholakia, Optical micromanipulation using a Bessel light beam, Opt. Commun. 197, 239 (2001).
[5] A. Boivin and E. Wolf, Electromagnetic Field in the Neighborhood of the Focus of a Coherent Beam, Phys. Rev. 138, B1561 (1965).

[6] L. C. Steinhauer and W. D. Kimura, A new approach for laser particle acceleration in vacuum, J. Appl. Phys. 72, 3237 (1992).

[7] E. Esarey, P. Sprangle, and J. Krall, Laser acceleration of electrons in vacuum, Phys. Rev. E 52, 5443 (1995).

[8] B. Hafizi, A. K. Ganguly, A. Ting, C. I. Moore, and P. Sprangle, Analysis of Gaussian beam and Bessel beam driven laser accelerators, Phys. Rev. E 60, 4779 (1999).

[9] Y. K. Ho, J. X. Wang, L. Feng, W. Scheid, and H. Hora, Electron scattering by an intense continuous laser beam, Phys. Lett. A 220, 189 (1996).

[10] J. X. Wang, Y. K. Ho, Q. Kong, L. J. Zhu, L. Feng, S. Scheid, and H. Hora, Electron capture and violent acceleration by an extra-intense laser beam, Phys. Rev. E 58, 6575 (1998).

[11] P. X. Wang, Y. K. Ho, X. Q. Yuan, Q. Kong, N. Cao, A. M. Sessler, E. Esarey, and Y. Nishida, Vacuum electron 
acceleration by an intense laser, Appl. Phys. Lett. 78, 2253 (2001).

[12] C. Harvey and M. Marklund, Radiation damping in pulsed Gaussian beams, Phys. Rev. A 85, 013412 (2012).

[13] L. Schächter and W. D. Kimura, Vacuum Channeling Radiation by Relativistic Electrons in a Transverse Field of a Laser-Based Bessel Beam, Phys. Rev. Lett. 114, 195501 (2015).

[14] J. Durnin, J. J. Miceli, and J. H. Eberly, Comparison of Bessel and Gaussian beams, Opt. Lett. 13, 79 (1988).

[15] B. C. Stuart, M. D. Feit, A. M. Rubenchik, B. W. Shore, and M. D. Perry, Laser-Induced Damage in Dielectrics with Nanosecond to Subpicosecond Pulses, Phys. Rev. Lett. 74, 2248 (1995).

[16] N. E. Andreev, S. S. Bychkov, V. V. Kotlyar, L. Y. Margolin, L. N. Pyatnitskii, and P. G. Serafimovich, Formation of high-power hollow Bessel light beams, Quantum Electron. 26, 126 (1996).
[17] J. Fan, E. Parra, I. Alexeev, K. Y. Kim, H. M. Milchberg, L. Ya. Margolin, and L. N. Pyatnitskii, Tubular plasma generation with a high-power hollow Bessel beam, Phys. Rev. E 62, R7603 (2000).

[18] P. M. Lapostolle, Possible emittance increase through filamentation due to space-charge effects, IEEE Trans. Nucl. Sci. 18, 1101 (1971).

[19] L. Schächter, Cerenkov traveling-wave tube with a spatially varying dielectric coefficient, Phys. Rev. A 43, 3785 (1991).

[20] N. M. Kroll, P. L. Morton, and M. N. Rosenbluth, Freeelectron lasers with variable wigglers, IEEE Quantum Electron. QE-17, 1436 (1981).

[21] I. M. Kapchinskij and V. V. Vladimirskij, Limitations of proton beam current in a strong focusing linear accelerator associated with the beam space-charge, in Proceedings of the International Conference on High Energy Accelerators (CERN, Geneva, 1959), p. 274. 